\title{
Vibrational Relaxation and Dissociation of Oxygen in Molecule-Atom Collisions
}

\author{
Daniil A. Andrienko* \\ Iain D. Boyd ${ }^{\dagger}$ \\ Department of Aerospace Engineering, University of Michigan, Ann Arbor, MI, 48109
}

\begin{abstract}
Collisions of the oxygen molecule with its parent atom are studied by the quasi-classical trajectory (QCT) method. A full set of VT and dissociation rates for the entire vibrational ladder is obtained utilizing an accurate $\mathrm{O}_{3}$ potential energy surface. Vibrational relaxation is studied for the ground electronic state of the $\mathrm{O}_{2}$ molecule at temperatures observed in hypersonic flows. The relaxation time is derived based on the removal rate for the first excited vibrational level. A significant deviation from the conventional formula by Millikan and White is observed for temperatures beyond those reported in experimental work. Vibrational relaxation in $\mathrm{O}_{2}-\mathrm{O}$ collisions becomes less efficient at high temperatures, suggesting that the efficiency of the energy randomization is strongly coupled to the attractive component of the $\mathrm{O}_{3}$ potential energy surface. These results are explained by analyzing the microscopic parameter of collisions that reflects the number of exchanges in the shortest interatomic distance. Under the conditions of strong energy randomization, the rates of rovibrational exothermic transitions are found to be nearly independent from the collision energy in the range of kinetic temperatures between 1,000 and 10,000 K. Curve fit coefficients are proposed for the updated vibrational relaxation time as well as for the state-specific dissociation rates.
\end{abstract}

\section{Nomenclature}

$\nu, j \quad$ vibrational and rotational quantum numbers

$E_{c o l}$ energy of collision, eV

$\tau_{v i b}$ vibrational relaxation time, $\mathrm{s}$

$P$ pressure, atm

$\Phi \quad$ transition probability

$b \quad$ impact parameter, $\stackrel{\AA}{ }$

$\sigma \quad$ cross section, $\AA^{2}$

$\mu \quad$ reduced mass, $\mathrm{g}$

$K \quad$ reaction rate, $\mathrm{cm}^{3} / \mathrm{s}$

$k_{B} \quad$ Boltzmann constant, erg/K

$g \quad$ degeneracy due to spin and angular momentum

Subscript

$\nu_{i}, \nu_{f}$ initial and final vibrational levels in bound-bound transitions

$j_{i}, j_{f}$ initial and final rotational levels in bound-bound transitions

$c \quad$ index of transition to continuum (dissociation)

\section{Introduction}

During the past several decades, significant progress has been achieved in hypersonic flight technologies. At the same time, it has become clear that further investigation is required in a manner where experiments

* Postdoctoral research fellow, Department of Aerospace Engineering, University of Michigan, 1320 Beal Ave

$\dagger$ James E. Knott Professor, Department of Aerospace Engineering, University of Michigan, 1320 Beal Ave 
and theoretical studies are coupled in order to provide reliable and repeatable results. In this sense, the improvement of existing models for simulating the complex aerothermodynamics is of interest for the present hypersonic research. One of the important aspects of hypersonic aerothemodynamics is the energy exchange among the internal degrees of freedom of species behind the shock wave. Since the translational mode can be excited much faster than vibrational, electron-electronic, and, at some conditions, rotational degrees of freedom, it is important to accurately model the energy exchange in order to predict the structure of the shock wave.

Among other phenomena that take place in the shock wave, vibrational excitation and deactivation play an important role in the energy balance. For the past decade, a significant improvement in the fidelity of existing models for air species was accomplished. High fidelity ab-initio calculations of potential energy surfaces (PES) and application of Monte-Carlo methods became available [1]. A full set of state-to-state cross-sectional data was obtained for the $\mathrm{N}_{2}-\mathrm{N}$ system under typical shock conditions. An attempt to calculate the dissociation rate of nitrogen under the assumption of a two-temperature model using the Quasi-Classical Trajectory (QCT) method has also been made [2].

Systems that contain oxygen are studied less often, mainly because oxygen quickly dissociates in a strong shock wave. However, during the flight at moderate hypersonic velocities, there is a significant amount of molecular oxygen in the post-shock region. This flight regime is inherent in hypersonic vehicles which travel at a speed of about $2 \mathrm{~km} / \mathrm{s}$. Oxygen chemistry is also important in the flight environment of scramjet engines.

The vibrational relaxation time of a significant number of diatomic species, many of which are encountered in hypersonic flows, is adequately described by the formula of [3] (M-W). However, there are several exceptions to this rule. The vibrational relaxation in NO-NO, CO-O, $\mathrm{O}_{2}-\mathrm{O}$ and $\mathrm{N}_{2}-\mathrm{O}$ collisions is several orders of magnitude faster than that of other species. Previously, the M-W equation was extended to all types of collisions in order to model thermal nonequilibrium in strong shock waves [4]. The relation between the macroscopic vibrational relaxation time, gas temperature and pressure, developed by Millikan and White, is based on the analysis of a large amount of experimental data and has the following form:

$$
\mathrm{P} \tau_{v i b}=\exp \left(\left(a \mathrm{~T}^{-1 / 3}-b\right)-18.42\right),
$$

where $\mathrm{P}$ is the pressure in Atmospheres, $\tau_{v i b}$ is the vibrational relaxation time in $\mathrm{s}, \mathrm{a}=0.00116 \mu^{1 / 2} \theta^{4 / 3}, \mathrm{~b}$ $=0.015 \mu^{1 / 4}, \theta$ is the characteristic vibrational temperature and $\mu$ is the reduced mass. Eq. (1) suggests that vibrational relaxation becomes more efficient when the gas temperature increases. Existing experimental studies of $\mathrm{O}_{2}-\mathrm{O}$ dynamics in a shock tube [5] were curve fitted by [4] and used to describe experimental data involving air ionization [6]. However, [7] stated that for the interaction of $\mathrm{O}_{2}$ and $\mathrm{O}$ a simple extrapolation of vibrational relaxation time beyond the experimental range of temperature may produce a significant error. Trajectory calculations, performed in [8], support the conclusion that the strong attraction in $\mathrm{O}_{2}-\mathrm{O}$ collisions plays an important role in the vibrational deactivation. Unfortunately, the reported theoretical data covers only a narrow temperature range and addresses deactivation rates only for certain vibrational levels. Thus, the theoretical investigation of thermal nonequilibrium in the $\mathrm{O}_{2}-\mathrm{O}$ system remains an area of interest.

A number of empirical and semi-empirical approaches have been proposed to model vibrational relaxation of air species. It is well known that the applicability of such models is satisfactory only in narrow ranges of temperatures. On the other hand, accurate quantum-mechanical models are available [9] at a very high computational cost. One can consider the quasi-classical trajectory method as a good compromise between accuracy and efficiency. The QCT method generates cross sections for atom-molecule collisions by applying the concept of Monte Carlo simulation and sampling of trajectories over the collision energies for either a specific rovibrational level or at some internal temperature. The QCT method was previously applied to obtain state-to-state rates for collisions between atom and molecule in high temperature air: [10], [11], [12]. The present paper focuses attention on the rovibrational energy relaxation in $\mathrm{O}_{2}-\mathrm{O}$ collisions.

This paper is organized as follows. The background studies of O2-O system are reviewed in Section II. The methodology adopted in the present work is discussed in Section III. The results are presented in Section IV. Finally, some conclusions are derived in Section V. The acknowledgments and appendix data are given in Sections VI and VII, respectively. 


\section{Background}

\section{A. Experimental studies of $\mathrm{O}_{2}-\mathrm{O}$ interaction}

The number of experimental studies of oxygen vibrational deactivation by collision with an oxygen atom is very limited. All papers can be divided into two categories depending on the reported temperature range. The first category presents data at atmospheric conditions [13,14]. Relaxation of molecular oxygen is observed through ozone photolysis by laser radiation. The reaction rates are usually limited to temperatures up to $350 \mathrm{~K}$. This experimental data may seem unimportant for hypersonic applications, however, since rovibrational transitions take place even at these temperatures, the cross-sectional data for low collision energies (less than $0.1 \mathrm{eV}$ ) has a certain influence on the region of moderate temperatures from 1,000 $\mathrm{K}$ to $5,000 \mathrm{~K}$. This fact is important due to the formation of a strong coupling complex by atomic and molecular oxygen at room temperature [7].

The second category of experimental studies reports results on $\mathrm{O}_{2}-\mathrm{O}$ collision dynamics obtained in shock tube facilities. To the authors' knowledge, only two experimental papers are available at the present time $[15,16]$. In the work by Kiefer and Lutz, the relaxation of oxygen was measured after the thermal dissociation of ozone in a shock wave. It was found that the relaxation time is several orders of magnitude smaller than in pure oxygen. This conclusion was explained by the presence of a strong chemical effect. The experimental data was then fit to a linear temperature dependence, as given by Eq. (2)

$$
\mathrm{P} \tau_{v i b}=(4.35 \pm 0.19) \times 10^{-8}-(7.75 \pm 0.81) \times 10^{-12} \mathrm{~T} \quad \text { atm } \cdot s, \mathrm{~T} \in 1600 \ldots 3300 \mathrm{~K}
$$

However, the technique adopted in [15] contains several important disadvantages. Because the methodology involves ozone, the concentration of atomic oxygen is subject to uncertainty since the rate of the exchange reaction involving $\mathrm{O}_{3}$ is not well established. Additionally, the thermal dissociation of ozone must occur much faster than $\mathrm{O}_{2}$ vibrational relaxation, and, thus, the temperature range is very limited. The later experimental measurements of vibrational relaxation time in $\mathrm{O}_{2}-\mathrm{O}$ mixtures were performed by Breen at al. [16]. Atomic oxygen was generated by a $\mathrm{RF}$ discharge in a $\mathrm{O}_{2}-\mathrm{Ar}$ mixture. It was found that the relaxation time could be fit by a linear temperature dependence, given by Eq. (3)

$$
\mathrm{P} \tau_{v i b}=(3.06 \pm 0.19) \times 10^{-8}-(2.18 \pm 8.34) \times 10^{-13} \mathrm{~T} \quad a t m \cdot s, \mathrm{~T} \in 1000 \ldots 3500 \mathrm{~K}
$$

Comparing Eqs. (2) and (3) one can notice a large difference in the temperature dependence. Breen's data indicates a much weaker dependence of the relaxation time with temperature. The uncertainty range in Eq. (3) allows the possibility for the vibrational relaxation time to increase with the gas temperature.

\section{B. Theoretical studies of $\mathrm{O}_{2}-\mathrm{O}$ interaction}

One of the first theoretical investigations of $\mathrm{O}_{2}-\mathrm{O}$ collision dynamics was by [7]. A vibrational deactivation rate was obtained by means of the adiabatic statistical channel model. Comparison of the "Napier" [17] vibrational relaxation time with the existing experimental data $[5,16]$ revealed a difference of the order of two to three times, increasing at high temperature. The theoretical data in [7] is available only at certain temperatures, however, it was shown that the vibrational relaxation rate at room temperature is 3 to 8 times faster than in the range of temperature from 1,500 to 2,000 K. While the difference with experiment may be explained by the particular PES used in the calculations, it is certainly clear that the vibrational relaxation is more efficient at low temperatures. This conclusion contradicts the conventional formula, Eq. (1), by Millikan and White.

The study of internal energy transfer in $\mathrm{O}_{2}-\mathrm{O}$ collisions by means of the QCT method was performed in $[11,18]$. Calculated state-specific cross sections were averaged for each vibrational level, assuming transrotational equilibrium. Comparison with other theoretical calculations [19] for the same potential energy surface, revealed a good agreement in multiquantum vibrational deactivation rates as well as thermally averaged dissociation rate. However, no attempt was made to compare the vibrational deactivation rate to the existing experimental data.

QCT simulations of the $\mathrm{O}_{3}$ system were also performed in [20] and [21]. Ivanov et al. used a newly derived PES for the $\mathrm{O}_{3}$ system [22]. It was found that the $\mathrm{O}_{2}$ monoquantum deactivation rate is nearly two times lower than the rate predicted by [7] and [13]. It is interesting to note that the reported rate is only slightly lower than the rate of $\mathrm{O}_{3}$ complex formation in the range of temperatures from $100 \mathrm{~K}$ to 2,000 
$\mathrm{K}$. The novel feature introduced in [21] is a straightforward accounting for multiple $\mathrm{O}_{3}$ PESs. A total of nine PESs due to spin multiplicity were generated by using an $a b$-initio molecular orbital method. The rate of vibrational monoquantum deactivation is reported for temperatures of 2,000 and $7,000 \mathrm{~K}$ for reaction channels that follow different PESs. A satisfactory agreement was observed with experimental data by [16], however the direct extrapolation of the experimental results to a temperature of $7,000 \mathrm{~K}$ contradicts the conclusion made in [7]. One of the unresolved questions that was not addressed in [21] is the large difference in the rate of monoquantum activation for different PESs. According to [21] at a temperature of 4,000 K there is an order of magnitude difference between the rates for $1^{1} A^{\prime}$ and $2^{1} A^{\prime}$ PESs, while the work by [23] suggests that all channels have to be equally important at temperatures higher than $500 \mathrm{~K}$.

\section{Methodology}

\section{A. Potential energy surface}

\section{Hulbert-Hirshfelder PES}

In the present work two potential energy surfaces of different fidelity are adopted to study the $\mathrm{O}_{3}$ system. The first PES is obtained by adding the pairwise interaction for all atoms. Previously, the pairwise PES was successfully applied in the study of rovibrational relaxation of oxygen in collisions with argon [12]. This potential surface does not account for the many-body interaction, however, the results of the QCT simulation, being compared to that using the accurate PES, can demonstrate the influence of the three-body interaction term. The simple potential surface is obtained as follows:

$$
V_{O_{3}}\left(r_{A B} ; r_{B C} ; r_{A C}\right)=V_{O_{2}, A B}\left(r_{A B}\right)+V_{O_{2}, B C}\left(r_{B C}\right)+V_{O_{2}, A C}\left(r_{A C}\right),
$$

where the diatomic interaction, $V_{O_{2}}(r)$, is given by the Hulburt-Hirschfelder $(\mathrm{H}-\mathrm{H})$ potential:

$$
V_{O_{2}}(r)=D\left[\left(1-\exp \left(-a\left(r-r_{e}\right)\right)\right)^{2}+c a^{3}\left(r-r_{e}\right)^{3} \exp \left(-2 a\left(r-r_{e}\right)\right)\left(1+a b\left(r-r_{e}\right)\right)\right] .
$$

Coefficients $a, b$ and $c$ are determined by the spectroscopic constants of oxygen, listed in [24]. The simple PES supports a maximum of 36 vibrational states and 223 rotational levels and a total number of 5117 rovibrational states of $\mathrm{O}_{2}\left(X^{3} \Sigma_{g}^{-}\right)$. The energies $e_{v, 0}$ of the $\mathrm{O}_{2}$ molecule in the ground rotational state and the corresponding number of rotational levels for vibrational level $\nu$ are given in Table 1. Zero energy corresponds to the nuclei separated by the equilibrium distance.

\begin{tabular}{ccc}
$\nu$ & $j_{\max }$ & $e_{v, 0}, \mathrm{eV}$ \\
\hline 0 & 223 & $9.7624 \mathrm{E}-02$ \\
1 & 218 & $2.9059 \mathrm{E}-01$ \\
2 & 213 & $4.8064 \mathrm{E}-01$ \\
3 & 209 & $6.6779 \mathrm{E}-01$ \\
4 & 205 & $8.5207 \mathrm{E}-01$ \\
5 & 200 & $1.0335 \mathrm{E}+00$ \\
6 & 196 & $1.2121 \mathrm{E}+00$ \\
7 & 192 & $1.3879 \mathrm{E}+00$ \\
8 & 188 & $1.5608 \mathrm{E}+00$ \\
9 & 183 & $1.7310 \mathrm{E}+00$ \\
10 & 179 & $1.8983 \mathrm{E}+00$ \\
11 & 175 & $2.0629 \mathrm{E}+00$ \\
12 & 170 & $2.2246 \mathrm{E}+00$
\end{tabular}

\begin{tabular}{ccc}
$\nu$ & $j_{\max }$ & $e_{v, 0}, \mathrm{eV}$ \\
\hline 13 & 166 & $2.3835 \mathrm{E}+00$ \\
14 & 162 & $2.5396 \mathrm{E}+00$ \\
15 & 157 & $2.6927 \mathrm{E}+00$ \\
16 & 153 & $2.8431 \mathrm{E}+00$ \\
17 & 148 & $2.9905 \mathrm{E}+00$ \\
18 & 143 & $3.1350 \mathrm{E}+00$ \\
19 & 139 & $3.2765 \mathrm{E}+00$ \\
20 & 134 & $3.4150 \mathrm{E}+00$ \\
21 & 129 & $3.5505 \mathrm{E}+00$ \\
22 & 124 & $3.6829 \mathrm{E}+00$ \\
23 & 118 & $3.8121 \mathrm{E}+00$ \\
24 & 113 & $3.9382 \mathrm{E}+00$
\end{tabular}

\begin{tabular}{ccc}
$\nu$ & $j_{\max }$ & $e_{v, 0}, \mathrm{eV}$ \\
\hline 25 & 108 & $4.0610 \mathrm{E}+00$ \\
26 & 102 & $4.1805 \mathrm{E}+00$ \\
27 & 96 & $4.2966 \mathrm{E}+00$ \\
28 & 90 & $4.4094 \mathrm{E}+00$ \\
29 & 84 & $4.5186 \mathrm{E}+00$ \\
30 & 77 & $4.6242 \mathrm{E}+00$ \\
31 & 70 & $4.7262 \mathrm{E}+00$ \\
32 & 63 & $4.8244 \mathrm{E}+00$ \\
33 & 55 & $4.9189 \mathrm{E}+00$ \\
34 & 45 & $5.0094 \mathrm{E}+00$ \\
35 & 34 & $5.0959 \mathrm{E}+00$ \\
36 & 19 & $5.1783 \mathrm{E}+00$
\end{tabular}

Table 1: Rovibrational structure of $\mathrm{O}_{2}\left(X^{3} \Sigma_{g}^{-}\right)$, HH PES

\section{Varandas and Pais PES}

An accurate many-body $\mathrm{O}_{3}$ PES [25] is also adopted for the purpose of high fidelity simulation. This PES is obtained by the double many body expansion method and contains two- and three-body interaction terms. The potential energy surface by Varandas generates 47 vibrational levels and a maximum of 236 rotational levels for molecular oxygen in the ground electronic state. The total number of rovibrational states is 
equal to 6,245 , however, taking into account the nuclear spin statistics of a homonuclear molecule, the even numbered rotational levels for the $\mathrm{O}_{2}\left(X^{3} \Sigma_{g}^{-}\right)$state are forbidden. Since, during a bound-bound rovibrational transition, the symmetry of the initial and final states cannot change, transitions of the $\mathrm{O}_{2}$ molecule in the ground electronic state are only allowed between odd-numbered rotational levels [26]. Energies $e_{v, 0}$ of the $\mathrm{O}_{2}$ molecule in the ground rotational state and corresponding number of rotational levels for vibrational level $\nu$ are given in Table 2. When incorporated into the QCT code, the potential surface, developed by Varandas, requires approximately 8 to 10 times more computational resources than used with the simple PES.

\begin{tabular}{ccc}
$\nu$ & $j_{\max }$ & $e_{v, 0}, \mathrm{eV}$ \\
\hline 0 & 236 & $9.7453 \mathrm{E}-02$ \\
1 & 231 & $2.9056 \mathrm{E}-01$ \\
2 & 226 & $4.8121 \mathrm{E}-01$ \\
3 & 221 & $6.6933 \mathrm{E}-01$ \\
4 & 217 & $8.5485 \mathrm{E}-01$ \\
5 & 213 & $1.0376 \mathrm{E}+00$ \\
6 & 209 & $1.2177 \mathrm{E}+00$ \\
7 & 205 & $1.3950 \mathrm{E}+00$ \\
8 & 201 & $1.5693 \mathrm{E}+00$ \\
9 & 196 & $1.7407 \mathrm{E}+00$ \\
10 & 192 & $1.9091 \mathrm{E}+00$ \\
11 & 188 & $2.0743 \mathrm{E}+00$ \\
12 & 184 & $2.2364 \mathrm{E}+00$ \\
13 & 180 & $2.3952 \mathrm{E}+00$ \\
14 & 176 & $2.5507 \mathrm{E}+00$ \\
15 & 172 & $2.7028 \mathrm{E}+00$
\end{tabular}

\begin{tabular}{ccc}
$\nu$ & $j_{\max }$ & $e_{v, 0}, \mathrm{eV}$ \\
\hline 16 & 168 & $2.8514 \mathrm{E}+00$ \\
17 & 164 & $2.9964 \mathrm{E}+00$ \\
18 & 160 & $3.1378 \mathrm{E}+00$ \\
19 & 155 & $3.2755 \mathrm{E}+00$ \\
20 & 151 & $3.4094 \mathrm{E}+00$ \\
21 & 147 & $3.5394 \mathrm{E}+00$ \\
22 & 143 & $3.6654 \mathrm{E}+00$ \\
23 & 138 & $3.7874 \mathrm{E}+00$ \\
24 & 134 & $3.9053 \mathrm{E}+00$ \\
25 & 129 & $4.0189 \mathrm{E}+00$ \\
26 & 125 & $4.1281 \mathrm{E}+00$ \\
27 & 120 & $4.2329 \mathrm{E}+00$ \\
28 & 115 & $4.3332 \mathrm{E}+00$ \\
29 & 110 & $4.4288 \mathrm{E}+00$ \\
30 & 105 & $4.5196 \mathrm{E}+00$ \\
31 & 100 & $4.6055 \mathrm{E}+00$
\end{tabular}

\begin{tabular}{ccc}
$\nu$ & $j_{\max }$ & $e_{v, 0}, \mathrm{eV}$ \\
\hline 32 & 95 & $4.6864 \mathrm{E}+00$ \\
33 & 90 & $4.7621 \mathrm{E}+00$ \\
34 & 84 & $4.8324 \mathrm{E}+00$ \\
35 & 79 & $4.8972 \mathrm{E}+00$ \\
36 & 73 & $4.9564 \mathrm{E}+00$ \\
37 & 67 & $5.0098 \mathrm{E}+00$ \\
38 & 61 & $5.0571 \mathrm{E}+00$ \\
39 & 54 & $5.0981 \mathrm{E}+00$ \\
40 & 47 & $5.1328 \mathrm{E}+00$ \\
41 & 40 & $5.1609 \mathrm{E}+00$ \\
42 & 33 & $5.1824 \mathrm{E}+00$ \\
43 & 26 & $5.1974 \mathrm{E}+00$ \\
44 & 19 & $5.2065 \mathrm{E}+00$ \\
45 & 12 & $5.2109 \mathrm{E}+00$ \\
46 & 6 & $5.2126 \mathrm{E}+00$ \\
47 & 0 & $5.2129 \mathrm{E}+00$
\end{tabular}

Table 2: Rovibrational structure of $\mathrm{O}_{2}\left(X^{3} \Sigma_{g}^{-}\right)$, Varandas PES [25]

\section{B. QCT method}

The internuclear separation in the isolated oxygen molecule is initialized by the random sampling of oscillation phase based on the period of vibrational motion. Initial separation of the target and projectile particles is given by $15 \AA$. Hamilton's differential equations, described in [27], are solved by the AdamsMoulton method of $11^{\text {th }}$ order of accuracy. The impact parameter, $b$, is sampled with a step size of $0.1 \AA$. Every batch contains $2 \times 10^{3}$ trajectories. Analysis of the final state is performed according to [28]. Each trajectory is integrated with the error in the total energy not exceeding $10^{-4 \%}$. The trajectory is terminated after the distance between products exceeds the initial separation. Each such trajectory is then classified into one of three possible channels: non-reactive bound-bound transition, exchange reaction or dissociation (bound-free transition).

The probability, $\Phi$, of the state-specific transition $\left(\nu_{i}, j_{i}\right) \rightarrow\left(\nu_{f}, j_{f}\right)$, where $\left(\nu_{i}, j_{i}\right)$ and $\left(\nu_{f}, j_{f}\right)$ are initial and final rovibrational states, at a given collision energy $E_{c o l}$ is defined as follows:

$$
\Phi\left(E_{c o l}\right)=\frac{2}{b_{\max }^{2}} \int_{0}^{b_{\max }}\left(\frac{N_{\nu_{i} j_{i} \rightarrow \nu_{f} j_{f}}}{N}\right) b d b .
$$

The probability of the bound-free transition is calculated in the manner, similar to Eq. 6 . In Eq. (6), $b_{\max }$ is the impact parameter at which only elastic collisions are observed, $N_{\nu_{i} j_{i} \rightarrow \nu_{f} j_{f}}$ and $N$ are the number of trajectories with the desired transition and the total number of trajectories in the current batch of impact parameter, respectively. The cross section of either bound-bound or bound free transition is calculated as follows:

$$
\sigma\left(E_{c o l}, \nu_{i}, j_{i}\right)=\pi b_{\max }^{2} \Phi\left(E_{c o l}, \nu_{i}, j_{i}\right)
$$

where $\Phi$ is sampled over the five variables, besides already mentioned in Eq. (7): impact parameter $b$, initial azimuthal $\theta$ and polar $\phi$ orientation of the target molecule, initial orientation of the target molecule angular momentum $\eta$ and initial vibrational phase $\xi$ of the target molecule. The reaction probability is also defined by the final rovibrational state as well as by the channel of interest. The present calculations study dynamics of $\mathrm{O}_{2}-\mathrm{O}$ collisions in the range of collision energy from 0.001 to $30 \mathrm{eV}$. This range is covered by 
43 intervals of uneven length. Stratified sampling of the impact parameter is adopted to increase accuracy of statistical modeling. All rovibrational levels are modeled by taking into account the results of nearly $1.7 \times 10^{7}$ trajectories.

As the first step in the analysis of the reaction rate, trans-rotational equilibrium is assumed. In order to do so, the cross section of transition from the initial state $\nu_{i}, j_{i}$ to any final rotational state of the final vibrational level $\nu_{f}$ is computed as follows:

$$
\sigma\left(E_{c o l}, \nu_{i}, j_{i} \rightarrow \nu_{f}\right)=\sum_{j_{f}} \sigma\left(E_{c o l}, \nu_{i}, j_{i} \rightarrow \nu_{f}, j_{f}\right) .
$$

The rate of either bound-bound or bound-free transition at the gas temperature $T$ is calculated by integrating the corresponding cross sections:

$$
K\left(\nu_{i}, j_{i}, T\right)=\frac{8 \pi}{\sqrt{\mu_{A, B C}}}\left(2 \pi k_{B} T\right)^{-3 / 2} \int_{0}^{\infty} \sigma\left(E_{c o l}, \nu_{i}, j_{i}\right) E_{c o l} \exp \left(\frac{-E_{c o l}}{k_{B} T}\right) d E_{c o l} .
$$

For further analysis it is convenient to average the reaction rate over all rotational levels that belong to the initial vibrational state. The averaging procedure assumes that each rotational level $j_{i}$ contributes to the rate according to the Boltzmann factor $w$ :

$$
w\left(j_{i}\right)=\epsilon g_{e} g_{s}\left(2 j_{i}+1\right) \exp \left(-\left(e_{v_{i}, j_{i}}-e_{v_{i}, 0}\right) / k_{B} T\right),
$$

where $\epsilon$ is the symmetric factor of $\mathrm{O}_{2}, \mathrm{~g}_{e}$ and $\mathrm{g}_{s}$ are the degeneracy of the electronic state and the nuclear spin, respectively. The Boltzmann-averaged rates of the bound-bound $\left(\nu_{i} \rightarrow \nu_{f}\right)$ and bound-free $\left(\nu_{i} \rightarrow c\right)$ transition are derived using Eqs.(11) and (12):

$$
\begin{gathered}
K_{\nu_{i} \rightarrow \nu_{f}}=\frac{\sum_{j_{i}}\left(w_{j_{i}} \sum_{j_{f}} K_{\nu_{i}, j_{i} \rightarrow \nu_{f}, j_{f}}\right)}{\sum_{j_{i}} w_{j_{i}}} ; \\
K_{\nu_{i} \rightarrow c}=\frac{\sum_{j_{i}} w_{j_{i}} K_{\nu_{i}, j_{i} \rightarrow c}}{\sum_{j_{i}} w_{j_{i}}} .
\end{gathered}
$$

A full set of vibrational-translational relaxation rates is generated by sampling the initial rotational state according to the Boltzmann distribution at temperatures of 1,000, 2,000, 3,000, 5,000 and 10,000 K. Also, a complete set of rovibrationally resolved QCT calculations are performed for $\nu_{i}$ equal to 1,10 and 20 .

\section{Non-adiabatic effects}

To simplify the analysis of results, transitions from the ground to excited electronic states are not considered. This assumption allows an implementation of the QCT method with a single potential energy surface. The characteristic temperatures of electronic excitation are 11,393 and $18,986 \mathrm{~K}$ for the $a^{1} \Delta_{g}$ and $b^{1} \Sigma_{g}^{+}$ states of $\mathrm{O}_{2}$. In this sense, one may expect that the present rates of bound-bound and bound-free transitions are altered at high temperatures, since for the present QCT simulation, each trajectory is forced to stay on the potential surface of the ground electronic state, disregarding the probability of potential energy surface hopping. On the other hand, accounting for this effect would significantly increase the cost of the QCT method without influence in the range of temperature of interest, where transition $\nu_{i}=1 \rightarrow \nu_{f}=0$ plays the dominant role. It is of interest to account for electronic excitation of $\mathrm{O}_{2}$, however, this is left for the future investigation.

Nevertheless, the degeneracy due to the spin and orbital momentum of reactants should be taken into account. For the bound-bound transition during collision of $\mathrm{O}\left({ }^{3} P\right)$ and $\mathrm{O}_{2}\left({ }^{3} X_{g}^{-}\right)$, the degeneracy factor can be expressed in the form, given by [23]:

$$
\frac{1}{g_{B B}}=3\left(5+3 \exp \left(-\frac{227.6}{T}\right)+\exp \left(-\frac{325.9}{T}\right)\right)
$$

The degeneracy factor of the bound-bound transition is found as a ratio of the PES degeneracy to the degeneracy of reactants. Taking into account that the present $\mathrm{O}_{3}$ PES is non-degenerate, the total degeneracy for the reaction of bound-bound transition is given by Eq. (13). At high temperatures, the degeneracy factor 
asymptotically approaches a factor of $1 / 27$. This means that the reaction takes place on only one of the 27 possible surfaces.

The degeneracy of the dissociation reaction should be assessed in a different manner. The dissociation of molecular oxygen in a shock wave with a temperature above a few thousands of $\mathrm{K}$ is highly unlikely to proceed in the adiabatic manner. One of the ways to account for an alternative channel of dissociation from the excited electronic state is to assume equilibrium between lower vibrational levels of the excited electronic state and high vibrational levels of the ground state [29]. The contribution of dissociation from the excited electronic level should be added to the dissociation rate from the ground electronic level.

It is possible to calculate the degeneracy factor of the dissociation reaction for each rovibrational level of the ground electronic state individually [30]. In this case, the degeneracy is calculated by assuming only electronic levels with the minimum of energy below the energy of the current rovibrational level. The degeneracy of the bound-free transition for the level $(\nu, j)$ is then computed as follows:

$$
g_{B F, \nu, j}=1+\frac{\sum g^{E}}{g^{X}},
$$

where $g^{X}=3$ is the degeneracy of the ground electronic state, and $g^{E}$ is the degeneracy of the electronically excited state. The assumption of equilibrium between the vibrational levels of the ground and excited states is valid if the next condition is held:

$$
e_{\nu, j}^{X}>e_{\nu=0, j=0}^{E} .
$$

Assuming that Eq. (15) is held for every rovibrational level of the ground electronic state, one can obtain the degeneracy factor of the bound-free transition, originally proposed in [29]: $g_{B F}=16 / 3$.

\section{Trajectory analysis}

To gain insight into the process of vibrational relaxation, mutual permutations of nuclei and energy randomization in $\mathrm{O}_{2}-\mathrm{O}$ collisions are studied in detail. Following the work of [31], the simulation of each collision is carried out tracking the number of exchanges in the shortest interatomic distance (NESID). This parameter is calculated by analyzing the distance between each pair of atoms. When the projectile atom A and target molecule BC are significantly separated from each other, the minimal interatomic distance is $\mathrm{R}_{B C}$. When the atom approaches the molecule, one can expect that at some moment the minimal interatomic distance may become either $\mathrm{R}_{A C}$ or $\mathrm{R}_{A B}$. If this happens, the shortest interatomic distance is reassigned and the NESID value is increased by unity. The NESID can be equal to zero, indicating that the projectile atom approached the molecule with the minimum of interaction. A high value of NESID corresponds to intense intermolecular energy transfer, as will be shown later. It is worth to note, that the NESID parameter is a meaningful characteristic only for collisions with a target and projectile molecule attracted to each other. For systems with a repulsive potential, such as $\mathrm{O}_{2}-\mathrm{Ar}$, the NESID value is always zero. In the present work, the NESID is calculated for low- and high-lying initial rovibrational states. NESID is tracked for each batch of impact parameter as well to investigate the favored conditions of collisions. Finally, the NESID value is studied separately for the exchange and nonreactive channels of collision.

Examples of trajectories with large NESID are shown in Figs. 1 and 2 for collision energies of 0.1 and $3 \mathrm{eV}$, respectively. These trajectories correspond to the head-on (direct) collision mode with the impact parameter set to zero. In the first case, the monoquantum deactivation $\nu_{i}=1, j_{i}=29 \rightarrow \nu_{f}=0, j_{f}=3$ takes place with the NESID of 23 . The projectile particle deeply penetrates into the collisional complex with the minimal approach of $1.1 \AA$ and a large number of interatomic oscillations occur. Due to the strong attraction, a bond between the projectile and target can be formed, resulting into the exchange channel.

Despite the small probability to be entrapped by the potential well at high collision energy, the projectile can efficiently transfer energy even at a small number of mutual vibrations. At collision energy of $3 \mathrm{eV}$ the number of interatomic vibrations is generally smaller than at low-energy conditions, as will be shown later. However, for the trajectory shown in Fig. 2, the projectile particle approaches the diatomic molecule at a distance of $1.2 \AA$ and effectively transfers kinetic energy to the target, exciting it by several vibrational quanta with the NESID equal to two. 


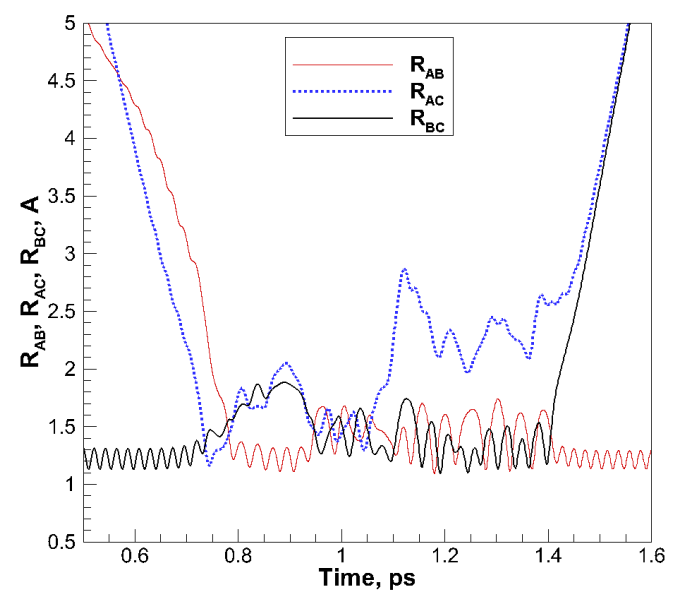

Fig. 1: Internuclear distance in $\mathrm{O}_{2}-\mathrm{O}$ collisions, $\nu_{i}=1, j_{i}=29 \rightarrow \nu_{f}=0, j_{f}=3$, collision energy $0.1 \mathrm{eV}, \mathrm{NESID}=23$

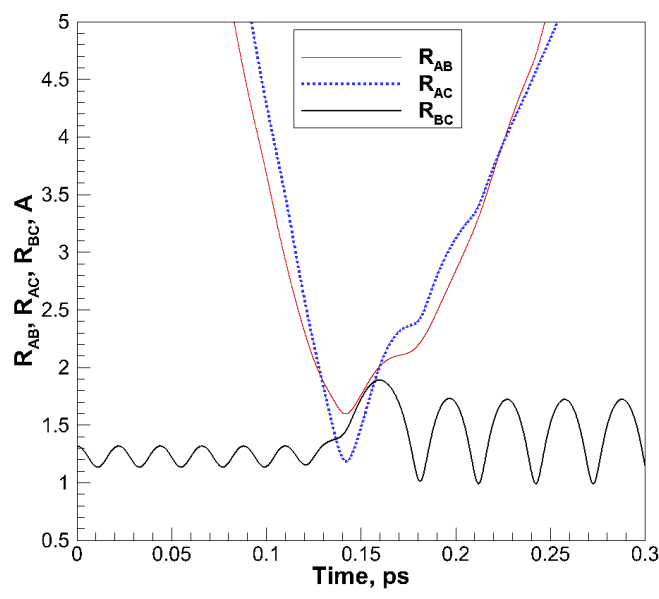

Fig. 2: Internuclear distance in $\mathrm{O}_{2}-\mathrm{O}$ collisions, $\nu_{i}=1, j_{i}=47 \rightarrow \nu_{f}=18, j_{f}=$ 27 , collision energy $3 \mathrm{eV}, \mathrm{NESID}=2$

\section{Results}

\section{A. Vibrational deactivation}

The first step in analysis of vibrational relaxation is the study of the deactivation rate between the first excited and ground vibrational levels. Since the populations of $\nu=0$ and $\nu=1$ account for at least 90 $\%$ of the entire vibrational ladder for temperatures below $2,000 \mathrm{~K}$, the two-state model provides important insight into the process of vibrational relaxation. The total cross section of monoquantum deactivation from rovibrational level $\nu_{i}=1, j_{i}$ to $\nu_{f}=0$, any $j_{f}$ is obtained by utilizing Eq. (8). The reaction rate of the process $\left(\nu_{i}, j_{i}\right) \rightarrow \nu_{f}$ is calculated using Eq. (9). The corresponding reaction rate $K_{\nu_{i}, j_{i} \rightarrow \nu_{f}}$ is averaged over the initial rotational levels $j_{i}$, assuming $T_{\text {rot }}=T$, to obtain the rate of monoquantum deactivation $K_{1 \rightarrow 0}$.

The monoquantum deactivation rates from $\nu_{i}=1$, obtained in the present work, are shown in Fig. 3. The solid line corresponds to the result, obtained using the Varadnas PES, the dashed line describes the $K_{1 \rightarrow 0}$ rate generated by the HH PES. Long dashed, dashed-dotted and double-dotted lines correspond to the results by Esposito [11], Quack [7] and Ivanov [20], respectively. Square symbols represent the QCT data by Matsukawa [21], triangles describe the experimental measurements [13,14]. Clearly, there is a significant dispersion in the previously reported data. The discrepancy is observed not only between experimental and theoretical results, but also between results obtained by the same QCT method. The rates shown by solid and long dashed curves are obtained using the same potential energy surface and degeneracy factor, given by Eq. (13). Results by [21], given by square symbols, show good agreement with present data at $\mathrm{T}=2,000$ $\mathrm{K}$, however at higher temperatures Matsukawa suggests much higher rates of deactivation.

The difference in $K_{1 \rightarrow 0}$, obtained using the $\mathrm{HH}$ and Varandas PESs, is due to the presence of three-body interaction in the $\mathrm{O}_{3}$ system. When the many-body repulsion is neglected, the QCT method predicts a higher rate of monoquantum deactivation. It is interesting to note that this difference vanishes at high temperatures, when particles interact mostly via the repulsive component of potential. For further investigation the most accurate results by the Varandas PES are considered, unless otherwise noted.

Results by [7] are in a fairly good agreement with the present data. The experimental measurements by [14] and [13] suggest twice lower rates of vibrational deactivation, however this disagreement applies only to room temperature that is not the focus of the present study. The results of [20] are clearly lower than the other data sets. Overall, the present QCT simulation suggests that the rate of monoquantum vibrational deactivation varies slowly with the temperature and is covered by the range of $10^{-11}-6 \times 10^{-12} \mathrm{~cm}^{3} / \mathrm{s}$ in the interval between $100 \mathrm{~K}$ and 10,000 K.

Rotationally resolved cross sections for the initial vibrational state $\nu_{i}=1$ are shown in Fig. 4 . Solid lines correspond to the cross section of the vibrational deactivation $\nu_{i}=1 \rightarrow \nu_{f}=0$, dashed lines describe the cross section of removal process: $\nu_{i}=1 \rightarrow \nu_{f} \neq 1$. The lowest allowed $j_{i}=1$ and highly excited $j_{f}=101$ rotational states are presented. One can see that, in the absence of a centrifugal barrier, the cross section of 


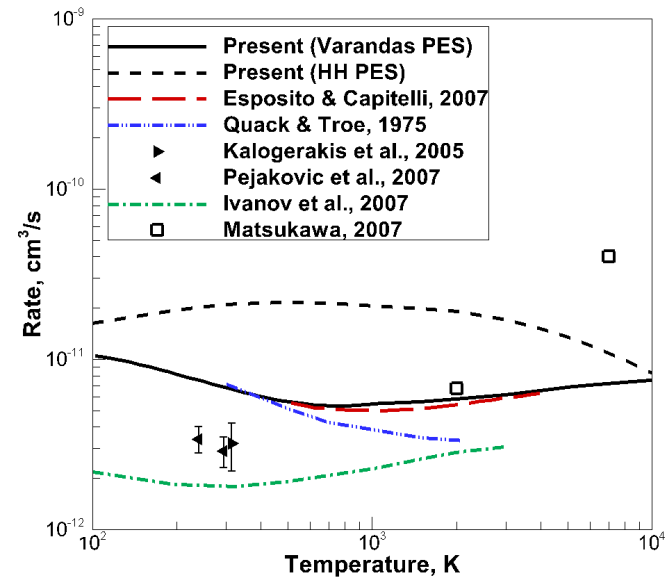

Fig. 3: Monoquantum vibrational deactivation rate from $\nu_{i}=1, \mathrm{~cm}^{3} / \mathrm{s}$

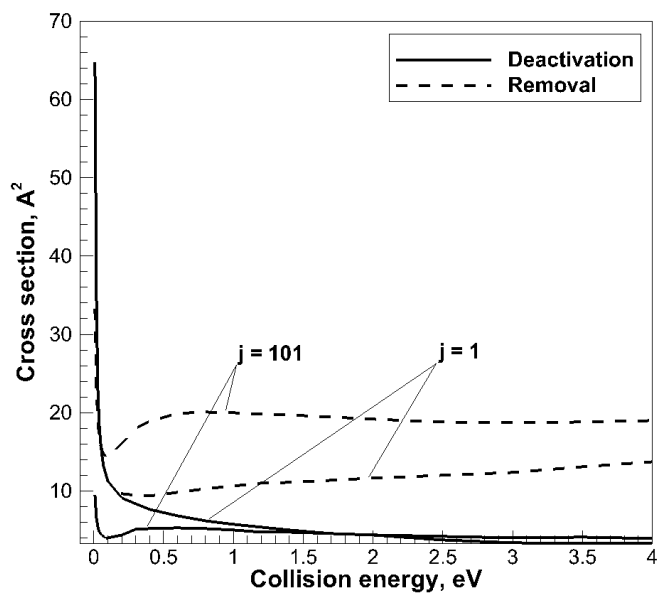

Fig. 4: Cross section of vibrational deactivation, Varandas PES

deactivation quickly increases at low collisional energies. This is mainly due to the fact that $\mathrm{O}_{2}-\mathrm{O}$ interaction is barrierless at a certain configuration of the colliding particles.

The vibrational excitation for $\left(\nu_{i}=1, j_{i}=1\right)$ starts to play a role at energies higher than $0.2 \mathrm{eV}$, which roughly corresponds to the energy threshold of $2275.5 \mathrm{~K}$ between $\nu=1$ and $\nu=2$. For the rapidly rotating oxygen molecule the cross section varies nonmonotonically at small collision energies due to the presence of the centrifugal barrier. However, the removal cross section is noticeably higher than that of vibrational deactivation, suggesting an efficient conversion of energy between internal degrees of freedom. This subject will be discussed later.

One of the most important parameters that can be extracted from the rate of monoquantum deactivation is the vibrational relaxation time. This parameter is best evaluated using numerical solution of the master equations for each rovibrational level, although this technique is computationally expensive. However, it is possible to obtain some qualitative results operating only with low lying vibrational levels. Assuming that the process of relaxation from the first excited to the ground vibrational level is the major contributor to the relaxation time, the latter can be expressed as follows:

$$
\mathrm{P} \tau_{v i b}=\frac{k_{B} T}{K_{1 \rightarrow 0}},
$$

where $K_{1 \rightarrow 0}$ is the rate of the $\nu_{i}=1 \rightarrow \nu_{f}=0$ transition. Also, considering both rates of vibrational activation and deactivation, it is possible to introduce the vibrational relaxation time that describes the total energy exchange between two vibrational levels:

$$
P \tau_{v i b}=\frac{k_{B} T}{K_{1 \rightarrow 0}+K_{0 \rightarrow 1}} .
$$

The "Napier" relaxation time, described by Eq. (17), was introduced in [17]. Experimental data, reported in [16], was also calculated using Eq. (17). Finally, vibrational relaxation time can be characterized by the total removal rate from $\nu_{i}=1$ :

$$
P \tau_{v i b}=\frac{k_{B} T}{\sum_{v \neq 1} K_{1 \rightarrow v}},
$$

Removal relaxation time, given by Eq. (18), was measured by [5]. Comparison of the present results with the experimental data is given by Fig. 5. The relaxation time computed from the QCT rates demonstrates a temperature dependence that is opposite to the Millikan-White expression, Eq. (1), presented by the solid curve with symbols. The present QCT simulation of $\mathrm{O}_{2}-\mathrm{O}$ collisions suggests that vibrational relaxation is very fast at low temperatures, and, as temperature increases, vibrational deactivation becomes less effective. At low temperatures the present relaxation time is in good agreement with both measurements by [5] and [16]. At high temperature limit, the "Napier" relaxation time deviates from the experimental measurements [16] 
by not more than $30 \%$. This agreement is found to be acceptable taking into account the overall dispersion in theoretical results, shown in Fig. 3. The disagreement of removal relaxation time with the data by [5] is larger, which can be explained by a large uncertainty in the measurements due to the rapid decomposition of ozone at high temperatures. Overall, the straightforward curve-fit of experimental data to the MillikanWhite expression leads to a severe overestimation of the relaxation time for temperatures below 2,000 K and to an underestimation in the high temperature region.

Comparison of vibrational relaxation time computed using Eqs.(16) and (18) with the available theoretical data is presented in Fig. 6. The rates of monoquantum deactivation based on the present simulation (solid line) and by Esposito (deltas) are in the excellent agreement. The relaxation time obtained from the removal rate deviates from the one obtained by Eq. (16) at temperatures higher than 1,000 K, indicating that vibrational excitation becomes active at these conditions. Results by [7], obtained using the statistical adiabatic channel model, also suggest a significant decrease of vibrational deactivation in the region of low temperatures. Relaxation times, calculated by Eq. (16) using the data from [13] and [14] are nearly twice larger than the present results, however this range of temperatures is not of interest for hypersonic simulations.

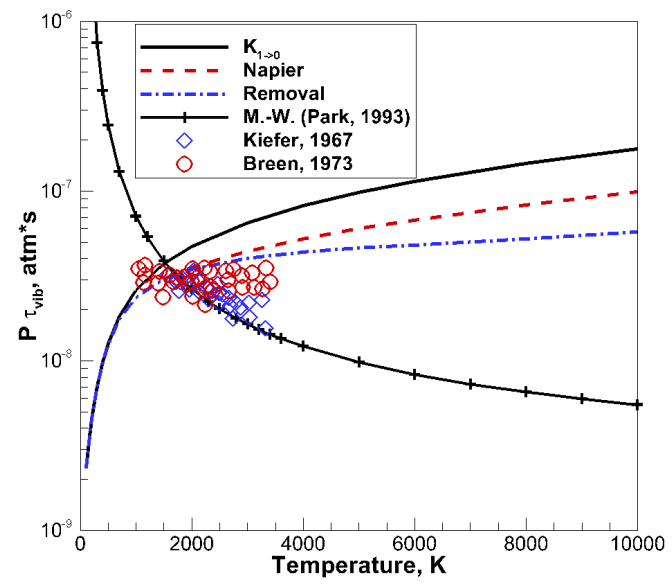

Fig. 5: Vibrational relaxation time: solid line - Eq. (16), dashed line - Eq. (17), dashed-dotted line - Eq. (18), o - [16], - [5], solid line with symbols - curve fit to [5] by [4]. Present data is obtained using Varandas PES

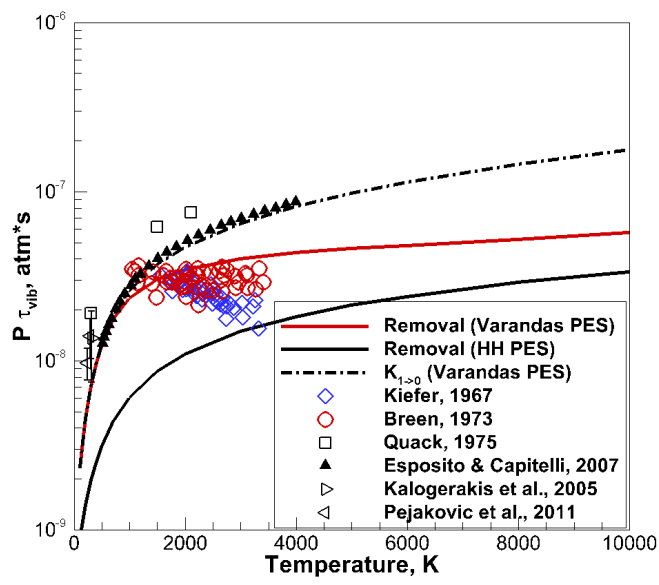

Fig. 6: Vibrational relaxation time: dashed-dotted line - Eq. (16), red solid line - Eq. (18) (Varandas PES), black solid line - Eq. (18) (HH PES), $\boldsymbol{\Lambda}$ - [18], Eq. (16), ○ $-[16], \diamond-[5], \triangleleft-[14], \triangleright-[13], \square-[7]$

The present relaxation time, obtain by Eq. (18) using an accurate many-body PES [25], can be fitted by a polynomial function of translational temperature in the interval between 1,000 and $10,000 \mathrm{~K}$ :

$$
P \tau_{v i b}=\left(a x^{4}+b x^{3}+c x^{2}+d x+e\right) \times 10^{-8}, A t m \times s
$$

where $x=T / 1000, T$ is in $\mathrm{K}, a=-0.001798, b=0.04853, c=-0.4695, d=2.143, e=0.673$. The root mean square error of the curve fit given by Eq. (19) is not more than $5 \times 10^{-10} \mathrm{Atm} \times \mathrm{s}$.

\section{B. Trajectory analysis}

In order to explain the increase of vibrational relaxation time with temperature, the trajectory dynamics are studied following the approach in [31]. The NESID value is measured for each computed trajectory at energies of $0.001,0.02,0.6$ and $3 \mathrm{eV}$. This range of collision energy is adopted to investigate the energy randomization at room temperature as well as under the post-shock conditions observed in hypersonic flows. Calculations are performed with the increment of impact parameter equal to $0.1 \AA$ and $\mathrm{N}=2 \times 10^{3}$ trajectories in each batch. The total number of trajectories depends on the collision energy and varies from $4.5 \times 10^{4}$ to $8.2 \times 10^{4}$ per collision energy per rovibrational state. Trajectory parameters are studied for both low and highly excited vibrational states $\left(\nu_{i}=1, j_{i}=1\right),\left(\nu_{i}=10, j_{i}=1\right)$ and $\left(\nu_{i}=20, j_{i}=1\right)$.

First of all, the monoquantum deactivation is studied for the rovibrational state $\left(\nu_{i}=1, j_{i}=1\right)$. The 
number of trajectories with the NESID in the range from 0 to 100 for transitions $\nu_{i}=1 \rightarrow \nu_{f}=1$ and $\nu_{i}=1 \rightarrow \nu_{f}=0$ is shown in Figs. 7 and 8, respectively. At collision energies of 0.001 and $0.02 \mathrm{eV}$, the number of trajectories leading to vibrational deactivation, is noticeably higher than the number of vibrationally elastic transitions. The amount of collisions leading to vibrational deactivation gradually decreases toward the high energy limit. At energies of 0.001 and $0.02 \mathrm{eV}$, the number of deactivation events at each NESID is nearly 3 to 4 times larger than the number of trajectories with no change of vibrational quantum number. As energy increases, vibrational relaxation becomes less efficient, and this difference vanishes. This can be clearly seen for the collision energy of $3 \mathrm{eV}$, when trajectories with or without change of vibrational quantum number have nearly the same value of NESID. At the same time, for either $\nu_{i}=1 \rightarrow \nu_{f}=0$ or $\nu_{i}=1 \rightarrow \nu_{f}=1$ transition, the number of trajectories with nonzero NESID quickly vanishes with increasing collision energy. This finding is in agreement with [31] at collision energies of $1-10 \mathrm{kcal} / \mathrm{mole}$ for the ground vibrational state.

Correlation of the NESID parameter and the probability of vibrational deactivation is even more evident for high vibrational states. The number of trajectories and the corresponding NESID for initial rovibrational state $\left(\nu_{i}=10, j_{i}=1\right)$ are given in Fig. 9 - 11. Transitions $\nu_{i}=10 \rightarrow \nu_{f}=10, \nu_{i}=10 \rightarrow \nu_{f}=9$ and $\nu_{i}=10 \rightarrow \nu_{f}<10$ are considered. The NESID parameter for initial state $\left(\nu_{i}=20, j_{i}=1\right)$ is shown in Figs. $12-14$.

For the multiquantum deactivation process, the number of trajectories with nonzero NESID in the range of collision energy from $0.001-0.6 \mathrm{eV}$ is at least an order of magnitude smaller than the number of vibrationally elastic collisions. The difference between the latter and a number of trajectories leading to monoquantum deactivation is smaller but still discernible. Overall, the number of trajectories with nonzero NESID as well as the average value of NESID reduces at higher initial vibrational quantum number. It is interesting to note that the number of trajectories leading to the multiquantum transition $\nu_{i}=10 \rightarrow \nu_{f}<10$ and $\nu_{i}=20 \rightarrow \nu_{f}<20$ is substantially higher than that for the transition $\nu_{i}=1 \rightarrow \nu_{f}=0$. This fact indicates that the process of vibrational deactivation in a chemically reactive system, such as $\mathrm{O}_{2}-\mathrm{O}$, occurs via large quantum jumps rather than by means of the ladder-climbing process [19].

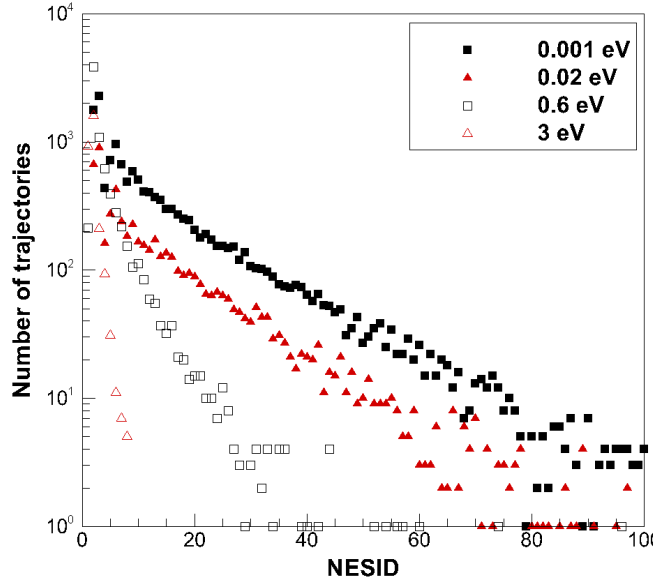

Fig. 7 : NESID, $\nu_{i}=1, j_{i}=1 \rightarrow \nu_{f}=1$

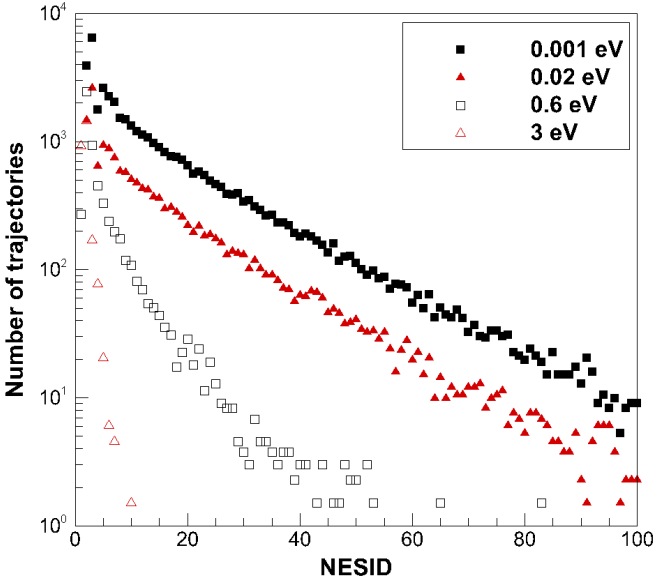

Fig. 8: NESID, $\nu_{i}=1, j_{i}=1 \rightarrow \nu_{f}=0$

The NESID strongly depends on the impact parameter of the collision, and, thus, on the potential energy surface, adopted for the QCT calculations. To demonstrate this fact, the NESID value is calculated at each batch of impact parameter for the initial state $\left(\nu_{i}=10, j_{i}=1\right)$. Results are shown by scatter diagrams in Figs. $15-17$ for collision energies of $0.02,0.6$ and $3 \mathrm{eV}$, respectively. For the sake of clarity, uninteresting trajectories with zero NESID are not shown.

At collision energy of $0.02 \mathrm{eV}$, the projectile particle has a large probability to be captured by the PES for the entire range of impact parameter. In this case, trajectories with nonzero NESID occur up to $b_{\max }$ of $6 \AA$ (not shown in Fig. 15). The NESID value is nearly independent of the impact parameter. As collision energy increases, $b_{\max }$ reduces down to $4.8 \AA$ and $4.6 \AA$ for the cases of 0.6 and $3 \mathrm{eV}$, respectively. At these energies, trajectories with nonzero NESID have a preferable impact parameter of 1.2 and $2.1 \AA$, as can be seen from Fig. 1. A projectile particle with such impact parameter is more likely to be captured by the potential well and induce an inelastic transition. The impact parameters of 1.2 and $2.1 \AA$ roughly 


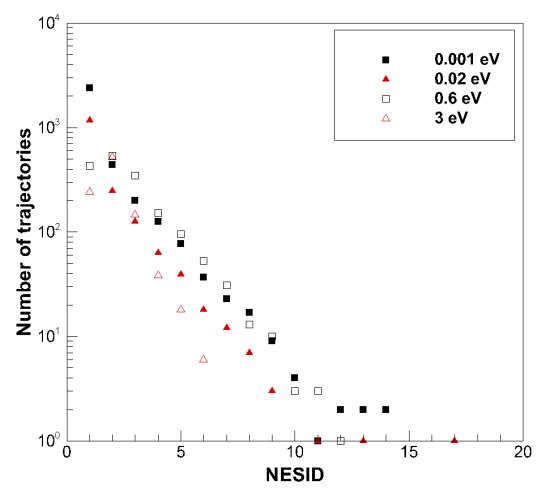

Fig. 9: NESID, $\nu_{i}=10, j_{i}=1 \rightarrow \nu_{f}=10$

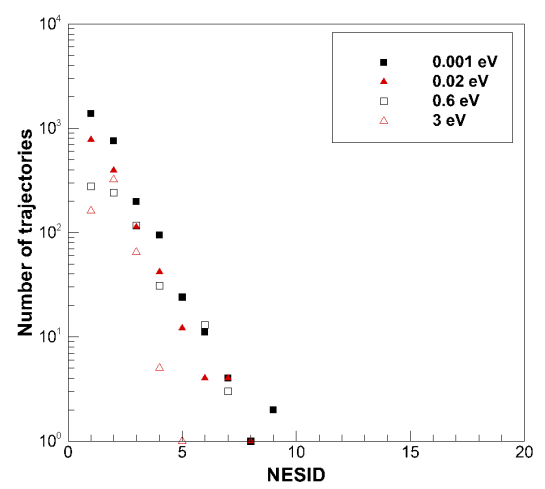

Fig. 12: NESID, $\nu_{i}=20, j_{i}=1 \rightarrow \nu_{f}=20$

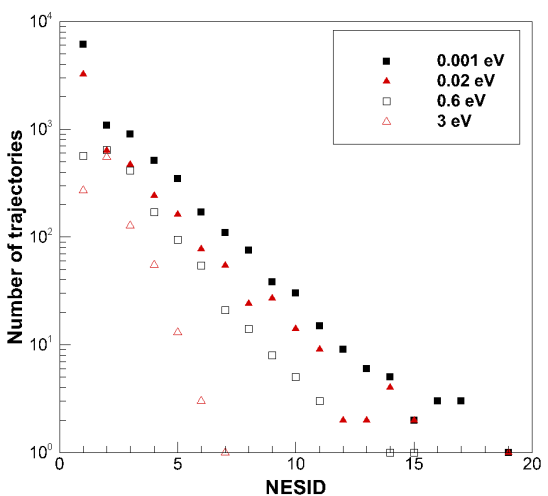

Fig. 10: NESID, $\nu_{i}=10, j_{i}=1 \rightarrow \nu_{f}=9$

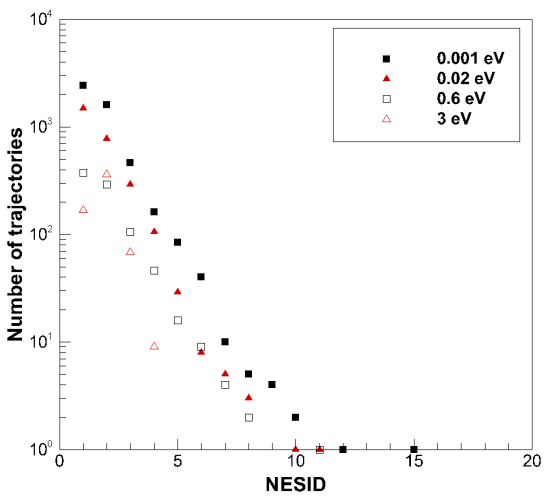

Fig. 13: NESID, $\nu_{i}=20, j_{i}=1 \rightarrow \nu_{f}=19$

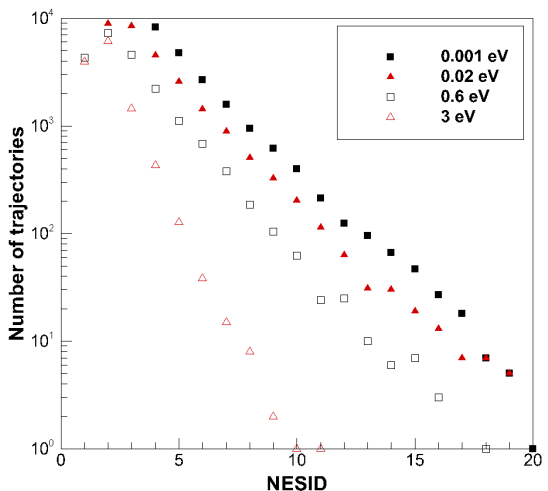

Fig. 11: NESID, $\nu_{i}=10, j_{i}=1 \rightarrow \nu_{f}<10$

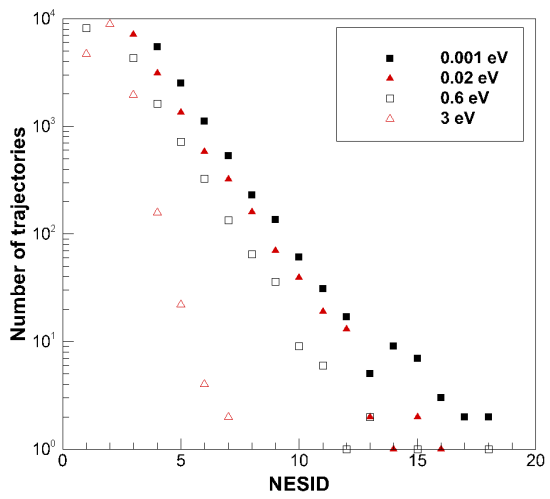

Fig. 14: NESID, $\nu_{i}=20, j_{i}=1 \rightarrow \nu_{f}<20$

correspond to the local minima of the Varandas PES in the collinear configuration of colliding particles. A trajectory analysis for $\nu_{i}=0$, performed in [31], revealed nearly the same favored values of impact parameter for the target molecule in a rotational state $j_{i}=11$.

The relative unimportance of impact parameter for the NESID value at low collision energies determines the cross section of vibrational deactivation at low temperatures. Since trajectories with higher NESID are more likely to produce vibrational deactivation, one may expect a more efficient interaction and, thus, the energy randomization between projectile and target particles at low collision energies. Moreover, the fact that the $b_{\max }$ is larger for low energies, indicates that the cross section of vibrational deactivation increases toward the low collisional energy limit, as is confirmed by Fig. 4.

\section{Internal energy exchange in target molecule}

One can expect that the $\mathrm{O}_{3}$ attractive potential affects not only the interaction between target and projectile particles but also the internal energy exchange in $\mathrm{O}_{2}$ induced by collision. As was mentioned above, the present implementation of the QCT method resolves state-specific rovibrational transitions. To investigate the energy transfer from the vibrational to the rotational mode during $\mathrm{O}_{2}-\mathrm{O}$ collision, the rates of deactivation from high vibrational state to the state with large $j$ are calculated. To study the inverse process, the rates of vibrational activation from highly excited rotational states are computed.

Figure 18 presents the state-specific deactivation rate for initial state $\left(\nu_{i}=10, j_{i}=1\right)$. Transitions with the change of vibrational quantum number $\Delta \nu=5$ and 1 are shown with symboled and plain lines, respectively. The state $\left(\nu_{i}=10, j_{i}=1\right)$ has the energy of $1.91 \mathrm{eV}$ and lies between the states $\left(\nu_{f}=9, j_{f}=21\right)$ and $\left(\nu_{f}=9, j_{f}=51\right)$ with the energies of 1.82 and $2.16 \mathrm{eV}$, respectively. From Fig. 18 it follows that the rate of exothermic transition $\left(\nu_{i}=10, j_{i}=1\right) \rightarrow\left(\nu_{f}=9, j_{f}=21\right)$ is nearly constant and does not depend on the 


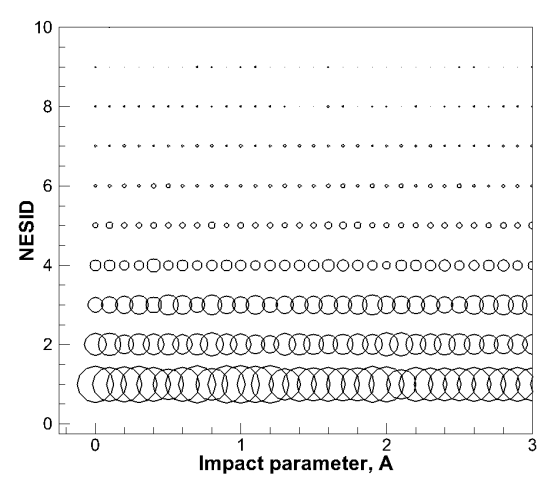

Fig. 15: $\left(\nu_{i}=10, j_{i}=1\right), 0.02 \mathrm{eV}$

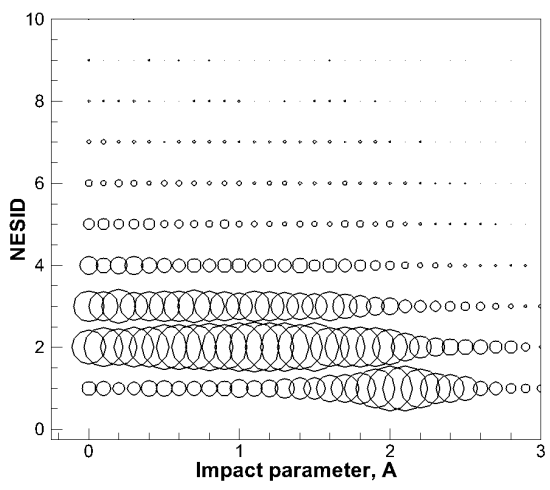

Fig. 16: $\left(\nu_{i}=10, j_{i}=1\right), 0.6 \mathrm{eV}$

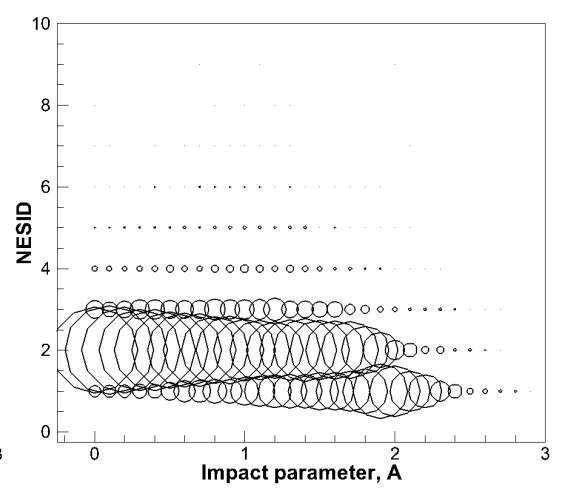

Fig. 17: $\left(\nu_{i}=10, j_{i}=1\right), 3 \mathrm{eV}$

thermal conditions. On the other hand, for the endothermic transition $\left(\nu_{i}=10, j_{i}=1\right) \rightarrow\left(\nu_{f}=9, j_{f}=51\right)$ the kinetic energy of the collision is a governing factor. In the case of $\Delta \nu=5$, the loss of vibrational energy is larger and transitions to highly excited rotational states become active at lower temperatures. The rates of endothermic transitions $\left(\nu_{i}=10, j_{i}=1\right) \rightarrow\left(\nu_{f}=5, j_{f}=21\right)$ and $\left(\nu_{i}=10, j_{i}=1\right) \rightarrow\left(\nu_{f}=5, j_{f}=51\right)$ are almost independent of the collision energy. Once the transition is energetically favored, the energy release due to vibrational deactivation is efficiently converted into the rotational energy of a lower vibrational state. The rate of such rovibrational transitions weakly depends on translational temperature.

For reference, the rates of the same transition, generated by the QCT method for the $\mathrm{O}_{2}-\mathrm{Ar}$ collision [12], are shown in Fig. 19. One can see that both endothermic and exothermic transition rates strongly depend on the thermal conditions. Note, that the absolute value of $\mathrm{O}_{2}-\mathrm{O}$ deactivation rates is generally much larger than that of $\mathrm{O}_{2}-\mathrm{Ar}$. The latter becomes comparable to the deactivation rate in the $\mathrm{O}_{3}$ system only at temperatures on the order of 10,000 K, when particles mostly interact via the repulsive branch of the potential. The comparison of Figs. 18 and 19 illustrates the influence of attractive and repulsive potentials on the energy randomization in a molecule-atom collision.

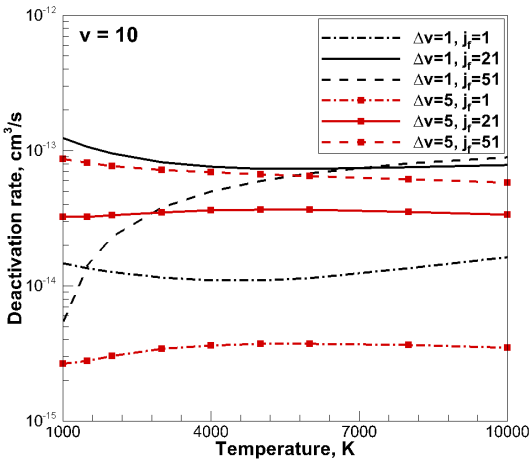

Fig. 18: $\mathrm{O}_{2}-\mathrm{O}$ deactivation rate from $\left(\nu_{i}=10, j_{i}=1\right)$

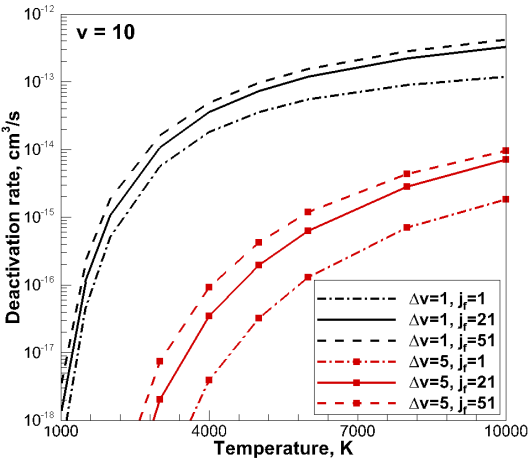

Fig. 19: $\mathrm{O}_{2}-\mathrm{Ar}$ deactivation rate from $\left(\nu_{i}=10, j_{i}=1\right)$

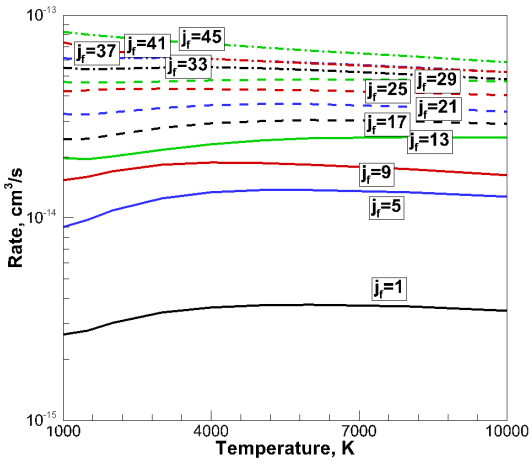

Fig. 20: $\mathrm{O}_{2}-\mathrm{O}$

rotationally-resolved deactivation rates, $\nu_{i}=10, j_{i}=1, \Delta \nu=5$

State-specific deactivation rates of transitions $\left(\nu_{i}=10, j_{i}=1\right) \rightarrow\left(\nu_{f}=5, j_{f}\right)$ are shown in Fig. 20. For a given change of vibrational energy, the rates of rovibrational transitions are nearly constant in the range of temperatures from 1,000 to 10,000 K and inversely proportional to the energy difference between the final and initial rovibrational states.

Energy conversion is also efficient for the process of vibrational activation from a high rotational state. For this purpose the following transitions are studied: $\left(\nu_{i}=1, j_{i}\right) \rightarrow\left(\nu_{f}, j_{f}=1\right)$, where $j_{i}$ takes a value of 21,51 or 101 and $\Delta \nu=1$ or 5 . The corresponding rates are shown in Fig. 21. Transition from the $\left(\nu_{i}=1, j_{i}=101\right)$ state is exothermic for all considered final states. Transitions with $j_{i}=51$ are endothermic only when $\Delta \nu=1$. Lastly, all transitions with $j_{i}=21$ are endothermic.

Similarly to the case of vibrational deactivation with a highly excited final rotational state, the rate 


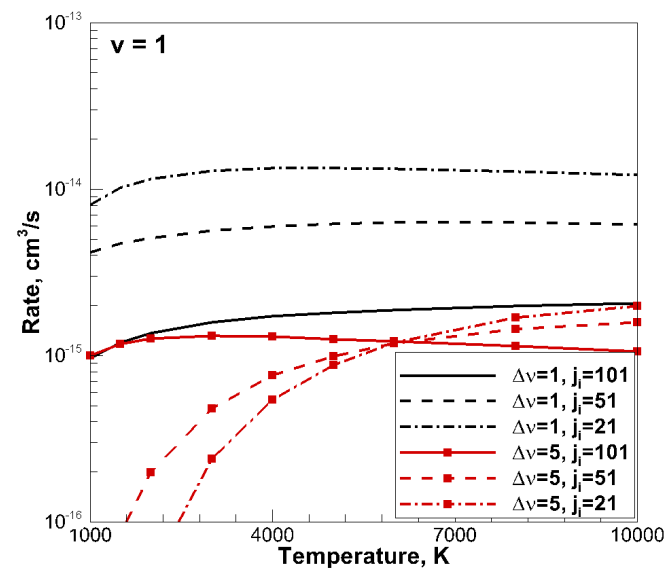

Fig. 21: $\mathrm{O}_{2}-\mathrm{O}$ rotationally-resolved activation rates, $\mathrm{cm}^{3} / \mathrm{s}, \nu_{i}=1, j_{i}=21,51$, 101

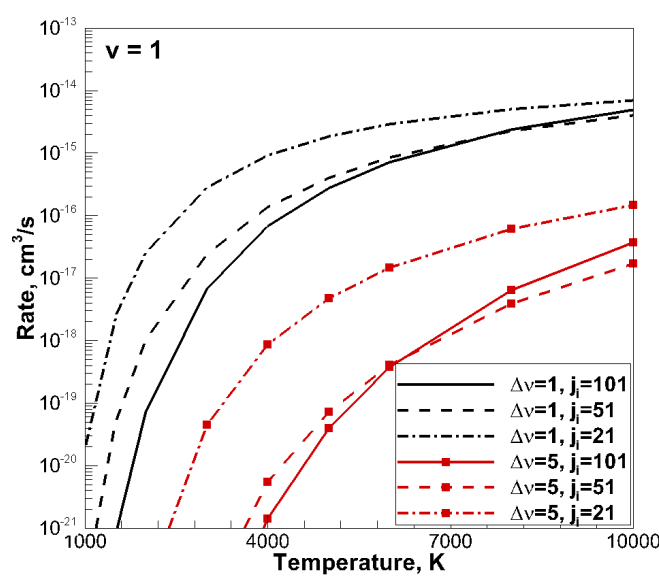

Fig. 22: $\mathrm{O}_{2}-\mathrm{Ar}$ rotationally-resolved activation rates, $\mathrm{cm}^{3} / \mathrm{s}, \nu_{i}=1, j_{i}=21,51$, 101

of vibrational activation of $\mathrm{O}_{2}$ by the parent atom weakly depends on temperature if such transition is energetically allowed, i.e. in the case of high initial rotational state. Moreover, if the transition does not require additional energy, the rate of vibrational activation is nearly the same for different $\Delta \nu$, as follows from Fig. 21 for the vibrational quantum jumps $\Delta \nu=1$ and $\Delta \nu=5$. This fact explains the large value of removal cross section for the target molecule at high initial rotational state, as can be seen in Fig. 4. On the other hand, vibrational excitation of oxygen by collision with the inert atom demonstrates a significant dependence on temperature, as can be seen in Fig. 22 for the collision of oxygen with argon. The rates of transitions with large $\Delta \nu$ are substantially lower than the corresponding rates for a single quantum jump.

\section{Exchange channel}

One of the important features, related to a chemically reactive system, such as $\mathrm{O}_{3}$, is a significant contribution of the exchange channel on the energy relaxation process. The influence of the reactive channel on rovibrational relaxation at different impact parameters is studied. Because the process of vibrational relaxation is tightly connected to the NESID value, investigation of the latter is performed for nonreactive and exchange channels at the following initial states of the target molecule: $\left(\nu_{i}=1, j_{i}=1\right),\left(\nu_{i}=10, j_{i}=1\right)$ and $\left(\nu_{i}=1, j_{i}=101\right)$. The particular interest in the relaxation dynamics of highly excited rovibrational states is due to its importance in hypersonic flows. The corresponding scatter diagrams are shown in Figs. 23 - 28 for the collision energy of $0.6 \mathrm{eV}$.

For the molecule in the low-lying initial rovibrational state, $\left(\nu_{i}=1, j_{i}=1\right)$ the direct collisions at small impact parameter mostly belong to the nonreactive channel. A distinguishable peak at $b \approx 2 \AA$ in Fig. 24 , at which the exchange channel contributes most, corresponds to the formation of a strongly coupled complex [31] and can be also observed for the process of vibrational deactivation in Figs. 16. The probability of exchange reaction increases with the rovibrational energy, as follows from Fig. 26 and 28. The favored impact parameter

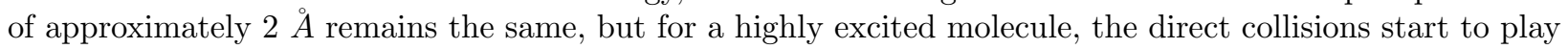
an important role. This is especially true for a target molecule with high initial rotational quantum number.

As was shown above, a collision with a rapidly rotating oxygen molecule is more likely to induce the removal of vibrational energy than when a molecule is in the ground rotational state. As follows from Fig. 4, the cross section of vibrational energy removal can be twice as large as the cross section of vibrational deactivation, even at very low kinetic energies. Contribution of the exchange and nonreactive channels during these transitions is shown in Figs. 29 and 30 for rotational temperatures of 1,000 and 10,000 K.

Overall, the cross section of the exchange channel diminishes at high collision energies for both deactivation and removal from $\nu_{i}=1$. At $\mathrm{T}_{r o t}=1,000 \mathrm{~K}$, the exchange channel is a dominant mechanism of vibrational relaxation at collision energies less than $0.1 \mathrm{eV}$. The contribution of the exchange channel at a rotational temperature of $10,000 \mathrm{~K}$ remains larger than that of the nonreactive channel for energies up to $0.4 \mathrm{eV}$. Note, that at high rotational temperature, the removal process is mostly due to the exchange chan- 


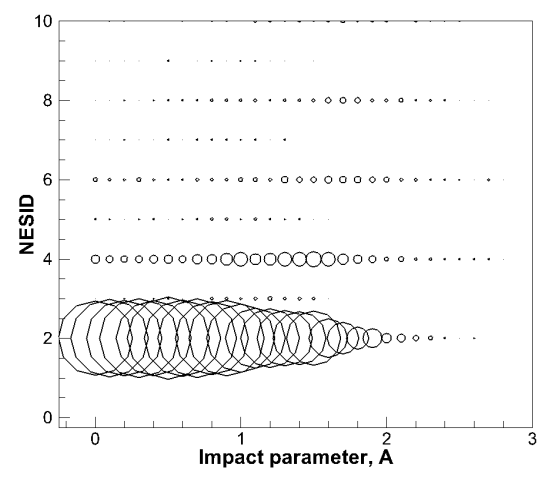

Fig. 23: Nonreactive channel, $\nu_{i}=1, j_{i}=1,0.6 \mathrm{eV}$

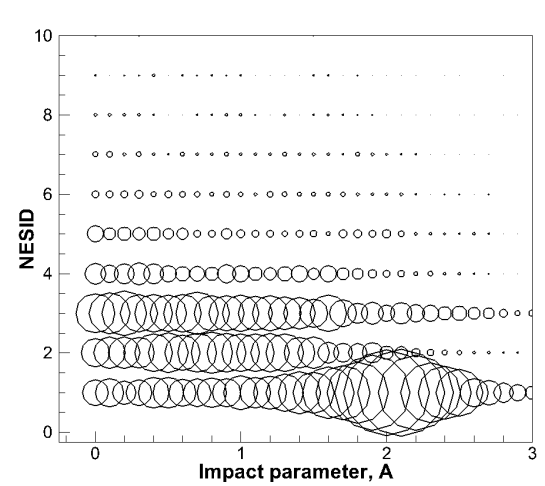

Fig. 26: Exchange channel, $\nu_{i}=10, j_{i}=1,0.6 \mathrm{eV}$

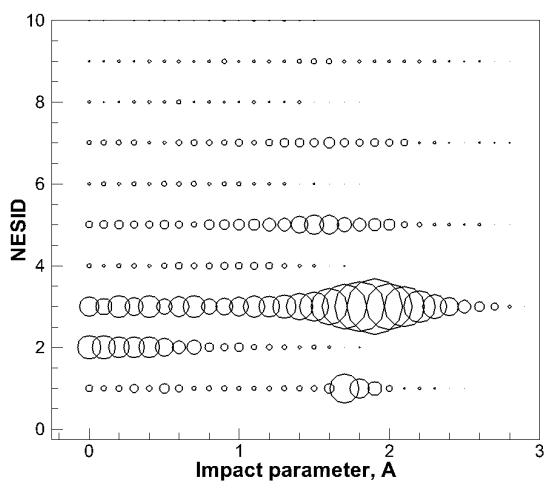

Fig. 24: Exchange channel, $\nu_{i}=1$, $j_{i}=1,0.6 \mathrm{eV}$

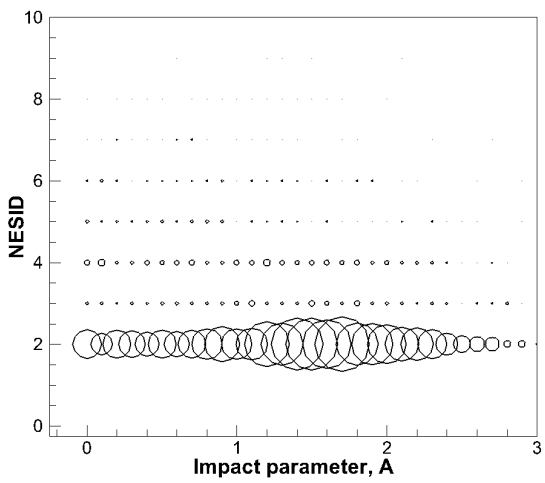

Fig. 27: Nonreactive channel, $\nu_{i}=1, j_{i}=101,0.6 \mathrm{eV}$

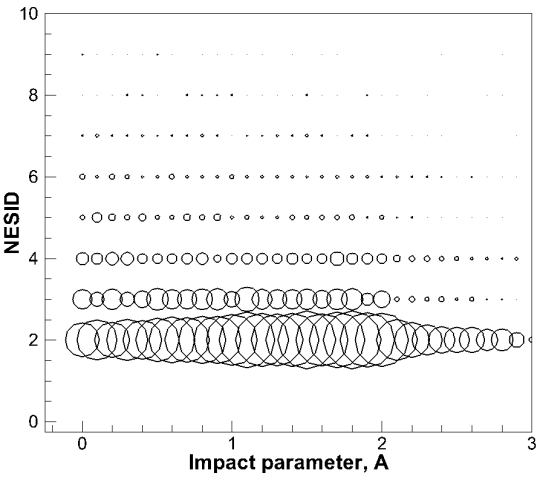

Fig. 25: Nonreactive channel, $\nu_{i}=10, j_{i}=1,0.6 \mathrm{eV}$

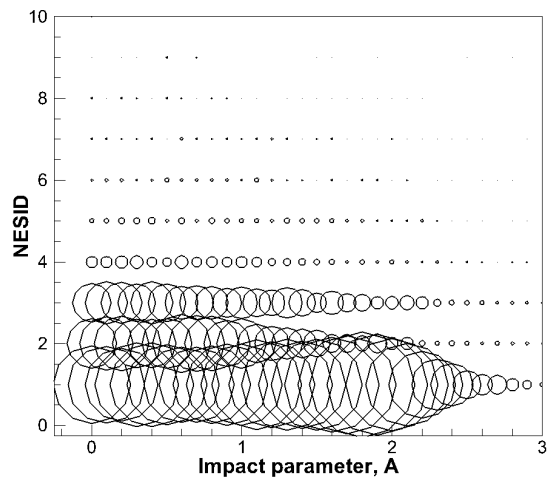

Fig. 28: Exchange channel, $\nu_{i}=1$, $j_{i}=101,0.6 \mathrm{eV}$

nel, while vibrational deactivation occurs mostly via the nonreactive channel of the $\mathrm{O}_{2}-\mathrm{O}$ collision. This is partially confirmed by the appearance of the scatter diagram in Fig. 28. One can expect an importance of the exchange channel not only for collisions with a molecule in a high initial rotational state, but also for the vibrationally excited target, as follows from Fig. 26.

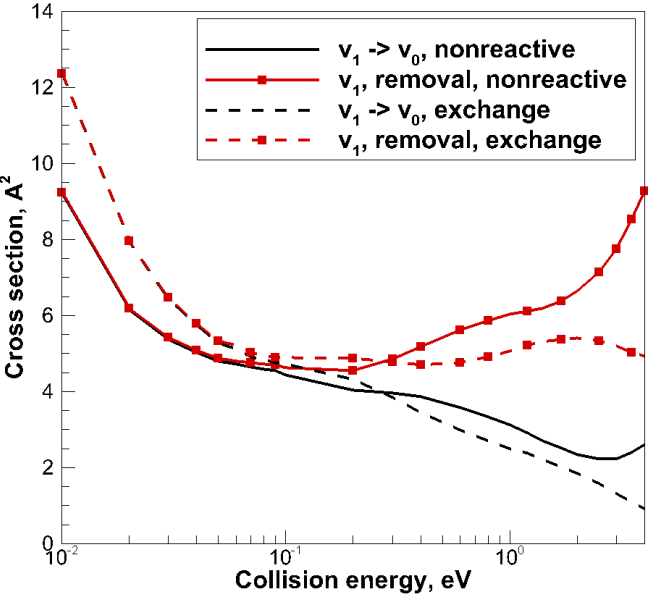

Fig. 29: $\sigma\left(\nu_{i}=1 \rightarrow \nu_{f}=0\right)$ and $\sigma\left(\nu_{i}=1 \rightarrow \nu_{f} \neq 1\right), \mathrm{T}_{\text {rot }}=1,000 \mathrm{~K}$

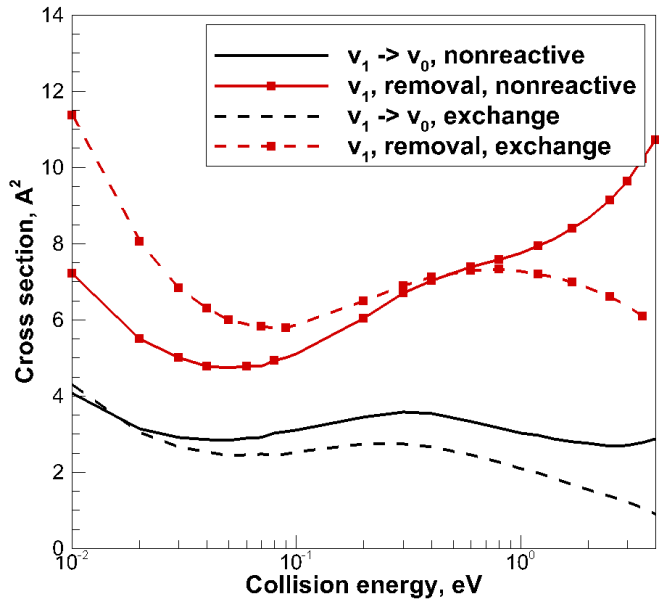

Fig. 30: $\sigma\left(\nu_{i}=1 \rightarrow \nu_{f}=0\right)$ and $\sigma\left(\nu_{i}=1 \rightarrow \nu_{f} \neq 1\right), \mathrm{T}_{\text {rot }}=10,000 \mathrm{~K}$ 


\section{E. Dissociation}

The present implementation of the QCT method is used to generate the state-specific dissociation rates in $\mathrm{O}_{2}-\mathrm{O}$ collisions. Oxygen quickly dissociates in strong shock waves and the depletion process has much less influence on the rovibrational temperature behind the shock wave than on the amount of atomic oxygen behind the shock wave [32]. Due to this reason, the study of oxygen dissociation by means of QCT method was performed only in a few works [18]. In the present, work vibrationally-resolved and vibrationally-averaged dissociation rates are obtained for the $\mathrm{HH}$ and Varandas potential energy surfaces. Generally speaking, it is incorrect to compare the state-specific rates for different PESs because of the different number of vibrational levels and their energies, especially close to the dissociation limit. However, the difference in the energy of low-lying vibrational levels does not exceed $0.2 \%$ between the PESs adopted in the present work. The comparison of state-specific dissociation rates with the results of Esposito [18] is performed for $\nu=10,20$, 30, 40 and for the last vibrational state, $\nu=46$ in Fig. 31.

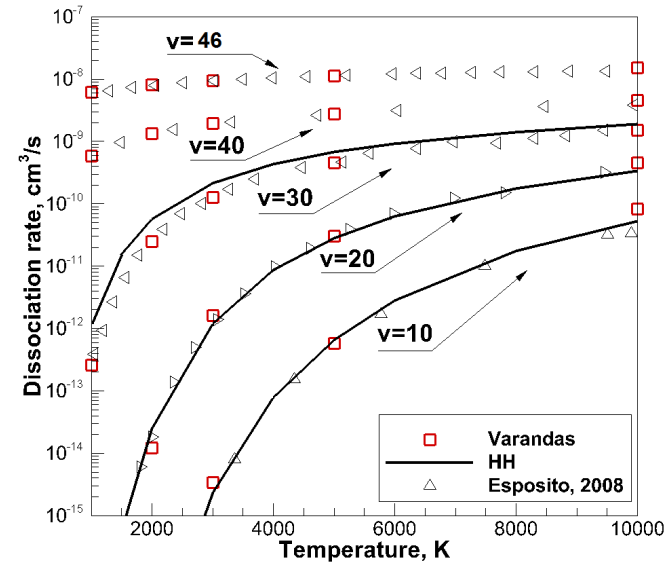

Fig. 31: State-specific dissociation rate, $\mathrm{HH}$ and Varandas PES

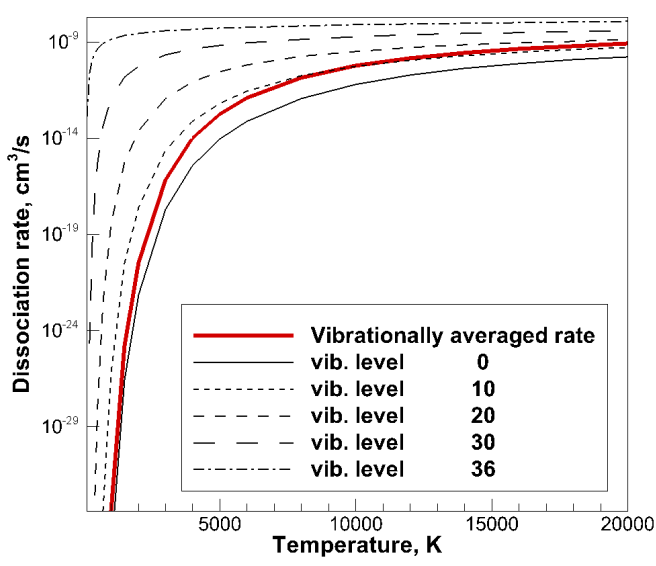

Fig. 32: Averaged dissociation rate, $\mathrm{HH}$ PES

The agreement in the state-specific dissociation rates between HH and Varandas PES is satisfactory for $\nu=10,20,30$. This fact is explained by the relative unimportance of the attractive component of potential for the dissociation channel. Agreement between the present rates via the Varandas PES and Esposito's rates is very good for the entire range of vibrational quantum number. There is a minor underestimation of the data by [11] at high temperatures due to the insufficient energy range adopted for the QCT simulations. The accurate state-specific dissociation rates, given by the Varandas PES, are curve-fitted to the generalized Arrhenius form in the range of temperatures from 1,000 to 20,000 K:

$$
\log \left(K_{d}(T, \nu)\right)=\log \left(A_{\nu}\right)+B_{\nu} \log (T)-\frac{C_{\nu}}{T},
$$

where $K_{d}$ is in $\mathrm{cm}^{3} / \mathrm{s}$, T is in K. Coefficients $A_{\nu}, B_{\nu}$ and $C_{\nu}$ for $\nu=0 \ldots 46$ are given in the Appendix.

Having in hand the full set of state-specific dissociation rates, it is possible to calculate the thermallyaveraged rate that accounts for the contribution of all vibrational levels at a given temperature. The selected state-specific rates and the averaged rate are shown in Fig. 32 for the HH PES. At low temperatures, the process of dissociation is governed by the low-lying vibrational levels. Specifically, the average rate closely follows the depletion rate from $\nu=1$ up to a temperature of 2,000 K. As the temperature increases, the dissociation rate is affected by excited vibrational levels. At the high temperature limit, the global dissociation rate approximately follows $K_{d}(T, \nu=20)$.

The thermally-averaged dissociation rate is in the satisfactory agreement with the data reported elsewhere $[4,32,33]$ as follows from Table 3. At low temperatures, the present QCT simulations, as well as Esposito's rates, suggests a smaller dissociation rate than was recommended previously [4], while at high temperatures the present results suggest a nearly $50 \%$ increase over the previously reported depletion rate [33].

The average dissociation rate can be easily curve-fitted to the generalized Arrhenius form as well. The present curve-fit parameters are reported for the reaction rate with the fixed adiabatic factor $g_{B F}=16 / 3$ in Table 4 . The activation energy is within $2 \%$ of the data, reported by different authors. The present 
results of the QCT simulation as well as the data by Esposito suggest a much smaller factor of temperature dependence as well as the pre-exponential constant compared with the previously reported data.

\begin{tabular}{cccccc}
\hline \hline Temperature, K & 1,000 & 2,000 & 5,000 & 10,000 & 20,000 \\
\hline Present, HH PES & $4.43 \times 10^{-34}$ & $4.36 \times 10^{-21}$ & $2.31 \times 10^{-13}$ & $6.96 \times 10^{-11}$ & $9.70 \times 10^{-10}$ \\
Present, Varandas PES & $5.94 \times 10^{-34}$ & $6.34 \times 10^{-21}$ & $2.92 \times 10^{-13}$ & $1.03 \times 10^{-10}$ & $1.49 \times 10^{-9}$ \\
Esposito & $7.04 \times 10^{-34}$ & $6.54 \times 10^{-21}$ & $2.99 \times 10^{-13}$ & $8.63 \times 10^{-11}$ & - \\
Park 1993 & $7.58 \times 10^{-33}$ & $2.23 \times 10^{-20}$ & $3.19 \times 10^{-13}$ & $4.33 \times 10^{-11}$ & $3.00 \times 10^{-10}$ \\
Dunn and Kang & $2.16 \times 10^{-33}$ & $8.98 \times 10^{-21}$ & $2.03 \times 10^{-13}$ & $3.89 \times 10^{-11}$ & $3.81 \times 10^{-10}$ \\
Shatalov & - & $4.14 \times 10^{-20}$ & $2.29 \times 10^{-13}$ & - & - \\
\hline \hline
\end{tabular}

Table 3: $\mathrm{O}_{2}+\mathrm{O} \rightarrow 3 \mathrm{O}$ reaction rate, $\mathrm{cm}^{3} / \mathrm{s}$

\begin{tabular}{cccc}
\hline \hline & $\mathrm{A}$ & $\mathrm{B}$ & $\mathrm{C}$ \\
\hline HH PES & $1.170 \times 10^{18}$ & -0.512 & 60650.0 \\
Varandas PES & $1.725 \times 10^{18}$ & -0.4037 & 60540.0 \\
Esposito & $3.73 \times 10^{18}$ & -0.557 & 60496.0 \\
Shatalov & $3.25 \times 10^{25}$ & -2.5 & 59380 \\
Park 1993 & $1.00 \times 10^{22}$ & -1.5 & 59500 \\
Dunn \& Kang & $9.00 \times 10^{19}$ & -1.0 & 59500 \\
\hline \hline
\end{tabular}

Table 4: Arrhenius parameters of dissociation reaction, pre-exponential factor is in $\mathrm{cm}^{3} / \mathrm{s} / \mathrm{mole}$

\section{Conclusion}

Vibrational relaxation in $\mathrm{O}_{2}-\mathrm{O}$ collisions is studied by means of the quasi-classical trajectory method using an accurate potential energy surface. The relaxation time is derived from the rate of vibrational energy removal from the first excited vibrational level. A significant discrepancy in the temperature dependence of the vibrational relaxation time between the present results and the conventional Millikan-White equation is found. Namely, the vibrational relaxation of oxygen by the parent atom is very fast at low collision energies and becomes less efficient as translational temperature increases. The analysis of the present QCT rates supports previously reported results about formation of a strongly coupled intermediate $\mathrm{O}_{3}$ complex. The efficiency of vibrational relaxation and energy randomization in $\mathrm{O}_{2}-\mathrm{O}$ collisions is tightly connected to the attractive part of the $\mathrm{O}_{3}$ potential energy surface.

The rates of vibrational relaxation, measured in a shock tube by Kiefer and Lutz and by Breen et al., are satisfactorily reproduced by the present approach in the range of temperatures from 1,000 to $3,600 \mathrm{~K}$. In the region of high temperature, where experimental measurements are not available, the present simulation suggests much slower vibrational relaxation than the Millikan-White relation. It is shown that the efficiency of vibrational relaxation is related to a number of exchanges in a shortest interatomic distance during a collision. Those trajectories leading to vibrational deactivation typically have substantially higher values of the NESID parameter. Such trajectories mostly occur at collision energies of $0.001-0.6 \mathrm{eV}$ because of the large contribution of the exchange channel. This behavior is more pronounced for molecules in a high initial vibrational state. The number of exchanges in the shortest interatomic distance decreases at high collision energies since the probability of the projectile particle to be entrapped by the potential well is reduced.

It was found that the rate of exothermic rovibrational transitions in $\mathrm{O}_{2}-\mathrm{O}$ collisions weakly depends on the collision energy and is nearly constant in the range of temperatures that are typically observed in hypersonic flows. In the present work, this phenomenon is associated with a strong energy randomization that takes place in the $\mathrm{O}_{3}$ complex. Such dependence of transition rates on kinetic temperature is noticeably different from the collision of oxygen with the inert atom in the presence of a repulsive potential. At the same 
time, a significant influence of the exchange channel on the process of vibrational relaxation was established at low collision energies and for oxygen molecules in a high rovibrational state.

The dissociation of oxygen is also studied by the QCT method. A good agreement with the previously reported data is established for both HH and Varandas potential energy surfaces. The present work reports Arrhenius curve fitting coefficients for the vibrationally-resolved dissociation rates obtained using the accurate three-body potential energy surface.

\section{Acknowledgments}

The authors gratefully acknowledge funding for this work through Air Force Office of Scientific Research Grant FA9550-12-1-0483. DA would also like to thank Dr. Jae Gang Kim for the $\mathrm{O}_{2}-\mathrm{Ar}$ QCT program code and numerous fruitful discussions.

\section{Appendix}

\begin{tabular}{|c|c|c|c|c|c|c|c|}
\hline$\nu_{i}$ & $\log \left(A_{\nu}\right)$ & $\mathrm{B}_{\nu}$ & C & $\nu_{i}$ & $\log \left(A_{\nu}\right)$ & $\mathrm{B}_{\nu}$ & $\mathrm{C}_{\nu}$ \\
\hline 0 & $-3572+01$ & $1.690+00$ & $5.92379+04$ & 23 & $-1.888+01$ & $-1.313-02$ & $2.05984+04$ \\
\hline U & $\begin{array}{l}-5.012+01 \\
-2.996+01\end{array}$ & $\begin{array}{l}1.090+00 \\
1.132+00\end{array}$ & $5.94163+04$ & 24 & $-1.840+01$ & $-6.379-02$ & $1.91373+04$ \\
\hline 2 & $-4.264+01$ & $2.398+00$ & $5.25105+04$ & 25 & $-2.649+01$ & 7.504-01 & $1.33478+04$ \\
\hline 2 & $-2.964+01$ & $1.103+00$ & $5.43525+04$ & 26 & $-2.055+01$ & $1.592-01$ & $1.52200+04$ \\
\hline 4 & $-2.696+01$ & $8.314-01$ & $5.33735+04$ & 27 & $-2.410+01$ & $5.037-01$ & $1.18825+04$ \\
\hline 5 & $-2.725+01$ & $8.578-01$ & $5.12154+04$ & 20 & -2.3 & $4.678-01$ & $1.07256+04$ \\
\hline 6 & $-3.074+01$ & $1.207+00$ & $4.71661+04$ & 29 & $-2.333+01$ & $4.236-01$ & $9.87797+03$ \\
\hline & $-2.931+01$ & $1.059+00$ & 4.5447 & 30 & $-2.387+01$ & $4.806-01$ & $8.41080+03$ \\
\hline 8 & $-2.962+01$ & $1.105+00$ & $4.2765 \overline{7}+04$ & 31 & $-2.406-$ & 4.969 & $2150+03$ \\
\hline 9 & $-2.434+01$ & $5.530-01$ & $4.27210+04$ & 32 & -2. & $5.096-01$ & $6.28916+03$ \\
\hline 10 & $-1.680+01$ & $-2.222-01$ & $4.30793+04$ & $\begin{array}{l}33 \\
34\end{array}$ & $\begin{array}{l}-2.4 \\
-2 \\
-2\end{array}$ & -01 & $\begin{array}{l}5.25579+03 \\
441984+03\end{array}$ \\
\hline 11 & -2.581 & -01 & $3.82245+04$ & 35 & $\begin{array}{l}-2.4505+01 \\
-2.425+01\end{array}$ & $5.359-01$ & $\begin{array}{l}4.41984+0.3 \\
3.66773+03\end{array}$ \\
\hline 12 & -2.5 & -01 & 3.6 & 36 & $-2.456+01$ & $5.676-01$ & $2.81714+03$ \\
\hline 14 & -2.2 & -01 & 3.5 & 37 & $-2.464+01$ & $5.816-01$ & $2.15376+03$ \\
\hline 15 & -2429 & $5.424-01$ & $36+04$ & $38+2+2$ & -2.42 & $5.586-01$ & $1.61583+03$ \\
\hline 16 & $-2.664+01$ & $7.758-01$ & $2.74940+04$ & 39 & -2.4 & -01 & $1.09847+03$ \\
\hline 17 & -1.733 & $-1.732-01$ & $2.97442+04$ & 40 & -2 & $5.827-01$ & $7.89248+02$ \\
\hline 18 & -2.302 & $083-01$ & $127+04$ & 49 & $-2.43 .5+01$ & $5.744-01$ & $\begin{array}{l}5.51008+02 \\
2\end{array}$ \\
\hline 10 & -1.92 & 175-02 & 2.55 & 43 & -2.349 & $5.175-01$ & $\begin{array}{l}2.99104+02 \\
1.35085+02\end{array}$ \\
\hline ק" & -2.075 & $00-01$ & $2.39310+04$ & 44 & $-2.197+c$ & 3.683-01 & $3.44300+02$ \\
\hline & & & 14 & & & $4.356-01$ & 5.59554-03 \\
\hline & & & & $40+2>3$ & -2.16 & $4.006-01$ & $4.35713-05$ \\
\hline
\end{tabular}

Table 5: Curve fit coefficients of state-specific dissociation rates

\section{References}

\footnotetext{
${ }^{1}$ R. Jaffe, D. Schwenke, and G. Chaban, "Theoretical analysis of $\mathrm{N}_{2}$ collisional dissociation and rotation-vibration energy transfer," in 47th AIAA Aerospace Sciences Meeting Including The New Horizons Forum and Aerospace Exposition, AIAA paper 2009-1569, 2009.

${ }^{2}$ J. Bender, I. Nompelis, P. Valentini, T. Schwartzentruber, G. V. Candler, S. Doraiswamy, Y. K. Paukku, K. R. Yang, Z. Varga, and D. G. Truhlar, "Quasiclassical trajectory analysis of the $\mathrm{N}_{2}+\mathrm{N}_{2}$ reaction using a new ab initio potential energy surface," in 11th AIAA/ASME Joint Thermophysics and Heat Transfer Conference, AIAA Paper 2014-2964, 2014.

${ }^{3}$ R. C. Millikan and D. R. White, "Systematics of vibrational relaxation," The Journal of Chemical Physics, vol. 39, no. 12 , p. $3209,1963$.

${ }^{4}$ C. Park, "Review of chemical-kinetic problems of future nasa missions. I - Earth entries," Journal of Thermophysics and Heat Transfer, vol. 7, no. 3, pp. 385-398, 1993.

${ }^{5}$ J. H. Kiefer and R. W. Lutz, "The effect of oxygen atoms on the vibrational relaxation of oxygen," in Symposium (International) on Combustion, vol. 11, pp. 67-76, Elsevier, 1967.

${ }^{6}$ J. Wilson, "Ionization rate of air behind high-speed shock waves," Physics of Fluids (1958-1988), vol. 9, no. 10, pp. 19131921,1966

${ }^{7} \mathrm{M}$. Quack and J. Troe, "Complex formation in reactive and inelastic scattering: Statistical adiabatic channel model of unimolecular processes III," Berichte der Bunsengesellschaft für physikalische Chemie, vol. 79, no. 2, pp. 170-183, 1975.

${ }^{8}$ E. L. Breig, "Statistical model for the vibrational deactivation of molecular by atomic oxygen," The Journal of Chemical Physics, vol. 51, no. 10, p. 4539, 1969.
} 
${ }^{9}$ V. Aquilanti, S. Cavalli, and D. De Fazio, "Hyperquantization algorithm. I. theory for triatomic systems," The Journal of chemical physics, vol. 109, no. 10, pp. 3792-3804, 1998.

${ }^{10} \mathrm{D}$. Bose and G. V. Candler, "Thermal rate constants of the $\mathrm{N}_{2}+\mathrm{O} \rightarrow \mathrm{NO}+\mathrm{N}$ reaction using abinitio3a and 3a potential energy surfaces," The Journal of chemical physics, vol. 104, no. 8, pp. 2825-2833, 1996.

${ }^{11}$ F. Esposito and M. Capitelli, "The relaxation of vibrationally excited $\mathrm{O}_{2}$ molecules by atomic oxygen," Chemical physics letters, vol. 443, no. 4, pp. 222-226, 2007.

${ }^{12}$ J. G. Kim and I. D. Boyd, "State-resolved master equation analysis of thermochemical nonequilibrium of nitrogen," Chemical Physics, vol. 415, pp. 237-246, 2013.

${ }^{13}$ K. S. Kalogerakis, R. A. Copeland, and T. G. Slanger, "Collisional removal of $\mathrm{O}_{2}\left(b^{1} \sum_{g}^{+}, \nu=2,3\right)$," Journal of Chemical Physics, vol. 116, pp. 4877-4885, 2002.

${ }^{14}$ D. A. Pejaković, Z. Campbell, K. S. Kalogerakis, R. A. Copeland, and T. G. Slanger, "Collisional relaxation of $\mathrm{O}_{2}$ $\left(X^{3} \sum_{g}^{-}, \nu=1\right)$ and $\left(a^{1} \Delta_{g}, \nu=1\right)$ by atmospherically relevant species," The Journal of chemical physics, vol. 135, no. 9, pp. -, 2011.

${ }^{15}$ R. W. Lutz and J. H. Kiefer, "Structure of the vibrational relaxation zone of shock waves in oxygen," Physics of Fluids (1958-1988), vol. 9, no. 9, pp. 1638-1642, 1966.

${ }^{16} \mathrm{~J}$. Breen, R. Quy, and G. Glass, "Vibrational relaxation of $\mathrm{O}_{2}$ in the presence of atomic oxygen," The Journal of Chemical Physics, vol. 59, no. 1, pp. 556-557, 1973.

${ }^{17}$ G. M. Burnett and A. M. North, "Transfer and storage of energy by molecules," Zeitschrift fr Physikalische Chemie, vol. 228,1969 .

${ }^{18}$ F. Esposito, I. Armenise, G. Capitta, and M. Capitelli, "O+O $\mathrm{O}_{2}$ state-to-state vibrational relaxation and dissociation rates based on quasiclassical calculations," Chemical Physics, vol. 351, pp. 91-98, Jul 2008.

${ }^{19}$ A. Lagana, A. Riganelli, G. Ochoa de Aspuru, E. Garcia, and M. Martinez, "On multiquantum vibrational deexcitation in symmetric reactions," Chemical physics letters, vol. 288, no. 5, pp. 616-620, 1998.

${ }^{20} \mathrm{M}$. Ivanov, R. Schinke, and G. McBane, "Theoretical investigation of vibrational relaxation of $\mathrm{NO}\left({ }^{2} \Pi\right), \mathrm{O}$, and $\mathrm{N}$ in collisions with $\mathrm{O}\left({ }^{3} \mathrm{P}\right), "$ Molecular Physics, vol. 105, no. 9, pp. 1183-1191, 2007.

${ }^{21}$ Y. Matsukawa, "Vibrational relaxation of oxygen by $\mathrm{O}_{2}-\mathrm{O}$ collision," Transactions of the Japan Society for Aeronautical and Space Sciences, vol. 49, no. 166, pp. 231-238, 2007.

${ }^{22}$ R. Siebert, P. Fleurat-Lessard, R. Schinke, M. Bittererová, and S. Farantos, "The vibrational energies of ozone up to the dissociation threshold: Dynamics calculations on an accurate potential energy surface," The Journal of chemical physics, vol. 116, no. 22, pp. 9749-9767, 2002.

${ }^{23}$ A. Gross and G. D. Billing, "Isotope effects on the rate constants for the processes $\mathrm{O}_{2}+\mathrm{O} \rightarrow \mathrm{O}+\mathrm{O}_{2}$ and $\mathrm{O}_{2}+\mathrm{O}+$ $\mathrm{Ar} \rightarrow \mathrm{O}_{3}+\mathrm{Ar}$ on a modified ground-state potential energy surface for ozone," Chemical physics, vol. 217, pp. 1-18, 1997.

${ }^{24}$ D. Steele, E. R. Lippincott, and J. T. Vanderslice, "Comparative study of empirical internuclear potential functions," Reviews of Modern Physics, vol. 34, no. 2, p. 239, 1962.

${ }^{25}$ A. Varandas and A. Pais, "A realistic double many-body expansion (DMBE) potential energy surface for ground-state $\mathrm{O}_{3}$ from a multiproperty fit to ab initio calculations, and to experimental spectroscopic, inelastic scattering, and kinetic isotope thermal rate data," Molecular Physics, vol. 65, pp. 843-860, Nov 1988.

${ }^{26}$ G. Herzberg, Molecular spectra and molecular structure. van Nostrand, 1957.

${ }^{27}$ R. B. Bernstein, "Atom-molecule collision theory; a guide for the experimentalist," in Atom-molecule Collision Theory; a Guide for the Experimentalist, vol. 1, 1979.

${ }^{28}$ D. G. Truhlar and J. T. Muckerman, "Reactive scattering cross sections III: quasiclassical and semiclassical methods," in Atom-Molecule Collision Theory, pp. 505-566, Springer, 1979.

${ }^{29}$ E. Nikitin, Theory of elementary atomic and molecular processes in gases. Oxford, Clarendon Press, 1974.

${ }^{30} \mathrm{~F}$. Esposito and M. Capitelli, "Quasiclassical trajectory calculations of vibrationally specific dissociation cross-sections and rate constants for the reaction $\mathrm{O}+\mathrm{O}_{2}=3 \mathrm{O}$," Chem. Phys. Lett., vol. 364, pp. 180-187, 2002.

${ }^{31}$ C. Lee and H.-R. Kim, "A classical trajectory study of $\mathrm{O}+\mathrm{O}_{2}$ collision," Chemical physics letters, vol. 233, no. 5, pp. 658-664, 1995.

${ }^{32}$ L. Ibraguimova, A. Sergievskaya, and O. Shatalov, "Dissociation rate constants for oxygen at temperatures up to 11000 k," Fluid Dynamics, vol. 48, no. 4, pp. 550-555, 2013.

${ }^{33}$ M. G. Dunn and S. Kang, "Theoretical and experimental studies of reentry plasmas," tech. rep., NASA-CR-2232, 1973. 